\title{
Inhibition of 2-hydroxyglutarate elicits metabolic reprogramming and mutant IDH1 glioma immunity in mice
}

\author{
Padma Kadiyala, ${ }^{1,2}$ Stephen V. Carney, ${ }^{1,2}$ Jessica C. Gauss, ${ }^{1,2}$ Maria B. Garcia-Fabiani, ${ }^{1,2}$ Santiago Haase, ${ }^{1,2}$ Mahmoud S. Alghamri, ${ }^{1,2}$ \\ Felipe J. Núñez, ${ }^{1,2}$ Yayuan Liu, ${ }^{3}$ Minzhi Yu, ${ }^{3}$ Ayman Taher, ${ }^{1,2}$ Fernando M. Nunez, ${ }^{1,2}$ Dan Li, ${ }^{3}$ Marta B. Edwards, ${ }^{1}$ Celina G. Kleer, ${ }^{4}$ \\ Henry Appelman, ${ }^{4}$ Yilun Sun, ${ }^{5,6}$ Lili Zhao, ${ }^{6}$ James J. Moon, ${ }^{3,7,8}$ Anna Schwendeman, ${ }^{3,7}$ Pedro R. Lowenstein, ${ }^{1,2,7}$ \\ and Maria C. Castro ${ }^{1,2,7}$
}

'Department of Neurosurgery, University of Michigan Medical School, Ann Arbor, Michigan, USA. 2Department of Cell and Developmental Biology, University of Michigan Medical School, Ann Arbor, Michigan, USA. ${ }^{3}$ Department of Pharmaceutical Sciences, University of Michigan, Ann Arbor, Michigan, USA. ${ }^{4}$ Department of Pathology, University of Michigan Medical School, Ann Arbor, Michigan, USA. ${ }^{5}$ Department of Radiation Oncology, University of Michigan Medical School, Ann Arbor, Michigan, USA. ${ }^{6}$ Department of Biostatistics, University of Michigan Medical School, Ann Arbor, Michigan, USA. 'Biointerfaces Institute, University of Michigan Medical School, Ann Arbor, Michigan, USA. ${ }^{8}$ Department of Biomedical Engineering, University of Michigan, Ann Arbor, Michigan, USA.

\begin{abstract}
Mutant isocitrate dehydrogenase 1 (IDH1-R132H; mIDH1) is a hallmark of adult gliomas. Lower grade mIDH1 gliomas are classified into 2 molecular subgroups: 1p/19q codeletion/TERT-promoter mutations or inactivating mutations in $\alpha$-thalassemia/mental retardation syndrome X-linked (ATRX) and TP53. This work focuses on glioma subtypes harboring mIDH1, TP53, and ATRX inactivation. IDH1-R132H is a gain-of-function mutation that converts $\alpha$-ketoglutarate into 2-hydroxyglutarate (D-2HC). The role of D-2HG within the tumor microenvironment of mIDH1/mATRX/mTP53 gliomas remains unexplored. Inhibition of D-2HC, when used as monotherapy or in combination with radiation and temozolomide (IR/TMZ), led to increased median survival (MS) of mIDH1 glioma-bearing mice. Also, D-2HG inhibition elicited anti-mIDH1 glioma immunological memory. In response to D-2HC inhibition, PD-L1 expression levels on mIDH1-glioma cells increased to similar levels as observed in WT-IDH gliomas. Thus, we combined D-2HC inhibition/IR/TMZ with anti-PDL1 immune checkpoint blockade and observed complete tumor regression in $60 \%$ of $\mathrm{mIDH} 1$ glioma-bearing mice. This combination strategy reduced $\mathrm{T}$ cell exhaustion and favored the generation of memory $\mathrm{CD}^{+} \mathrm{T}$ cells. Our findings demonstrate that metabolic reprogramming elicits anti-mIDH1 glioma immunity, leading to increased MS and immunological memory. Our preclinical data support the testing of IDH-R132H inhibitors in combination with IR/TMZ and anti-PDL1 as targeted therapy for mIDH1/mATRX/mTP53 glioma patients.
\end{abstract}

\section{Introduction}

Gliomas are highly infiltrative brain tumors accounting for $32 \%$ of all primary central nervous system malignancies (1). With advances in molecular biology and sequencing technologies, a distinct profile of genetic alterations for gliomas has emerged (1-3). A gain-of-function mutation in the gene encoding isocitrate dehydrogenase 1 (IDH1) mutation has been reported in approximately $20 \%-25 \%$ of all infiltrative gliomas $(4,5)$. This mutation results in the replacement of arginine $(\mathrm{R})$ for histidine $(\mathrm{H})$ at amino acid residue 132 (R132H) $(6,7)$. In gliomas, the IDH1-R132H mutation co-occurs with the following genetic alterations: (a) $1 \mathrm{p} / 19 \mathrm{q}$ codeletion, and TERT promoter mutations in oligodendroglioma, or (b) inactivation of tumor suppressor protein 53 (TP53) gene and loss-of-function mutations in alpha thalassemia/mental retardation syndrome $\mathrm{X}$-linked gene $(A T R X)$ in astrocytoma $(2,8,9)$.

Conflict of interest: The authors have declared that no conflict of interest exists. Copyright: @ 2021, American Society for Clinical Investigation.

Submitted: May 11, 2020; Accepted: December 9, 2020; Published: February 15, 2021.

Reference information: J Clin Invest. 2021;131(4):e139542.

https://doi.org/10.1172/JCl139542.
The IDH1-R132H neomorphic mutation (mIDH1) confers a gain-of-function catalytic activity, prompting the NADPHdependent reduction of alpha ketoglutarate $(\alpha-K G)$ to the oncometabolite D-2-hydroxyglutarate (D-2HG) $(6,10,11)$. The accumulation of D-2HG acts antagonistically to $\alpha$-KG, competitively inhibiting $\alpha-\mathrm{KG}$-dependent dioxygenases, including the ten-eleven translocation (TET) methylcytosine dioxygenases and Jumonji C (JmjC) domain-containing histone demethylases (6, 11-13). This leads to a DNA and histone H3 hypermethylation phenotype, resulting in an epigenetic reprogramming of the glioma cell transcriptome $(9,14,15)$.

Ongoing research has demonstrated the benefits of targeting IDH1-R132H in gliomas with small molecule inhibitors (16). The compound AGI-5198 is an allosteric, competitive inhibitor that is selective for the IDH1-R132H enzyme, inhibiting the synthesis of D-2HG in mouse and human glioma cells $(7,15,16)$. Our laboratory generated a genetically engineered mouse model of glioma expressing IDH1-R132H (mIDH1) concomitantly with loss of ATRX and TP53 to study IDH1-R132H within the scope of the genetic lesions encountered in human astrocytomas $(2,9)$. Using this model, we demonstrated that AGI-5198 treatment decreased 
D-2HG levels, induced radiosensitivity, and decreased proliferation of mIDH1 glioma neurospheres in vitro (9). AGI-5198 has also been shown to inhibit D-2HG production in vivo and impair tumor growth in an anaplastic oligodendroglioma patient-derived xenograft model expressing 1p/19q codeletion and IDH1-R132H (17). AG-120, an IDH1-R132H inhibitor that is structurally related to AGI-5198, is currently being evaluated clinically for the treatment of cholangiocarcinoma (ClinicalTrials.gov NCT02989857) and acute myeloid leukemia (AML) (ClinicalTrials.gov NCT03173248) (18). Recently, AG-120 has been shown to have a favorable safety profile for nonenhancing IDH1-R132H gliomas (19).

Herein, we used the genetically engineered mouse model of glioma expressing IDH1-R132H and loss of ATRX and TP53 (mIDH1) (9) to elucidate the role played by D-2HG in the glioma immune microenvironment. Our findings demonstrate that treating mIDH1 glioma-bearing mice with IDH1-R132H inhibitor alone or in combination with ionizing radiation (IR) and temozolomide (TMZ), which is standard of care (SOC) for mIDH1 glioma patients, substantially prolonged the median survival (MS) of mIDH1 glioma-bearing mice and elicited antiglioma immunity. To date, the efficacy of PD-1/PD-L1 immune checkpoint blockade therapy has not been explored in glioma models harboring IDH1-R132H in the context of ATRX and TP53 loss. In this study, we demonstrate that coadministering $\alpha \mathrm{PD}-\mathrm{L} 1$ immune checkpoint blockade with IDH1-R132H inhibition and SOC markedly enhances the overall survival of mIDH1 gliomabearing mice. Furthermore, this treatment strategy reduces $\mathrm{T}$ cell exhaustion and promotes the generation of memory $\mathrm{CD}^{+} \mathrm{T}$ cells, leading to immunological memory. Collectively, our findings demonstrate that upon metabolic reprogramming it is possible to elicit anti-mIDH1 glioma immunity, leading to increased MS and antitumor immunological memory. Our data support the clinical testing of IDH1-R132H inhibitors in combination with SOC and $\alpha$ PD-L1 immune checkpoint blockade to treat glioma patients expressing IDH1-R132H in the context of TP53, ATRX inactivating mutations.

\section{Results}

Inhibition of D-2HG production sensitizes $m I D H 1$ glioma to radiotherapy and induce immunogenic cell death. In this study, we sought to determine whether inhibiting D-2HG production would sensitize mIDH1 glioma to IR treatment and induce immunogenic cell death (ICD). We performed a clonogenic assay on mIDH1 and WT-IDH mouse neurospheres (mouse-NSs) (Figure 1A) and human glioma cells (human-GCs), by treating them with the mIDH1 inhibitor AGI-5198 and IR. Compared with mIDH1 mouseNSs subjected to IR alone, we observed a significant reduction in clonogenic survival of mIDH1 mouse-NSs treated with AGI-5198 and IR ( $P \leq 0.0001)$, suggesting that $\mathrm{D}-2 \mathrm{HG}$ inhibition radiosensitizes mIDH1 NS (Figure 1B). On the other hand, we observed a dose-dependent decrease in clonogenic survival of WT-IDH mouse-NSs in response to IR, irrespective of AGI-5198 treatment (Figure 1C). Notably, treating radioresistant human mIDH1 glioma cells (MGG119), which endogenously express IDH1$\mathrm{R} 132 \mathrm{H}, A T R X$, and TP53 inactivating mutations, with AGI-5198 rendered them radiosensitive ( $P \leq 0.0001$; Figure 1D). AGI-5198 treatment of radiosensitive human WT-IDH (SJGBM2) cells, which endogenously express ATRX and TP53 inactivating mutations, did not alter their response to radiation (Figure 1E).

Next, we assessed if AGI-5198 therapy in combination with IR induces ICD in mIDH1 mouse-NSs and human-GCs. We measured the levels of calreticulin (CRT), ATP, HMGB1, IL-1 $\alpha$, and IL-6, common damage-associated molecular pattern molecules (DAMPs) expressed by dying tumor cells (20). First, we quantified the levels of CRT expression in mIDH1 mouse-NSs and humanGC cells in response AGI-5198 alone, IR alone, or AGI-5198+IR treatments. The mIDH1 mouse-NSs treated with AGI-5198 displayed a 6-fold $(P \leq 0.0001)$ increase in CRT expression relative to vehicle and IR alone groups (Figure $1 F$ ). This response was further increased by approximately 1.5 -fold $(P \leq 0.0001)$ with AGI5198+IR treatment (Figure 1F). A similar response was observed in mIDH1 human-GCs treated with AGI-5198+IR (Figure 1G).

We also assessed the amount of ATP released in the supernatants of mIDH1 mouse-NSs and human-GCs in response to AGI-5198, IR, or AGI-5198+IR treatments. We observed a 2-fold $(P \leq 0.01)$ increase in the extracellular release of ATP in the supernatant of mIDH1 mouse-NSs and human-GCs treated with AGI5198 compared with vehicle treatment (Figure $1 \mathrm{H}$ ). We observed an additional 1.5-fold $(P \leq 0.001)$ increase in extracellular ATP in the supernatant of mIDH1 mouse-NSs and human-GCs treated with AGI-5198+IR (Figure 1H). No difference was observed in ATP release between IR and AGI-5198 treatment groups in mIDH1 mouse-NSs. However, there was a 1 -fold $(P \leq 0.05)$ increase in ATP release in mIDH1 human-GCs treated with AGI-5198 compared with the IR treatment group (Figure $1 \mathrm{H}$ ).

It has been reported that tumor cells treated with radiotherapy release high levels of ATP, triggering autophagy activation (21-23). Microtubule-associated protein light chain 3 (LC3) is involved in the initiation of autophagosome formation. The protein exists in 2 forms: cytoplasmic LC3-I and membrane-bound LC3-II. The increase in LC3-II levels indicates autophagy flux activation. Western blot analysis revealed increased levels of LC3-II in mIDH1 mouse-NSs and human-GCs treated with IR alone compared with untreated controls (Supplemental Figure 1; supplemental material available online with this article; https:// doi.org/10.1172/JCI139542DS1).

Last, we tested the release of HMGB1 in the supernatants of mIDH1 mouse-NSs and human-GCs in response to AGI-5198, IR, or AGI-5198+IR treatments. We observed a 1.5-fold $(P \leq 0.001)$ increase in the extracellular release of HMGB1 in the supernatant of mIDH1 mouse-NSs treated with AGI-5198 compared with the IR or vehicle treatment groups (Figure 1I). We observed an additional 1.6-fold $(P \leq 0.01)$ increase in extracellular HMGB1 release in the supernatant of mIDH1 mouse-NSs with AGI-5198+IR (Figure 1I). A similar response was elicited in mIDH1 human-GCs treated with AGI-5198+IR (Figure 1I). We observed comparable results for additional DAMPs such as IL-1 $\alpha$ (20) and IL-6 (20) in both mIDH1 mouse-NSs and human-GCs (Supplemental Figure 2). Taken together, these results demonstrate that AGI-5198+IR induces ICD in mIDH1 mouse-NSs and human-GCs.

We performed flow cytometry analysis to determine the impact of IDH1-R132H in the context of ATRX and TP53 loss on the immune microenvironment of sleeping beauty (SB) gliomas and intracranial gliomas generated from SB-derived NS. 
A

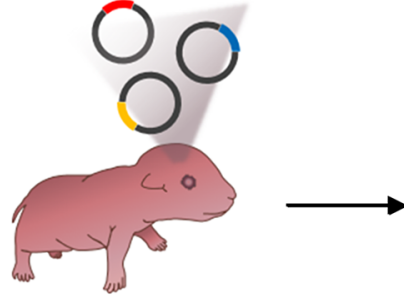

Plasmid DNA injection into the lateral ventricle of neonate mice

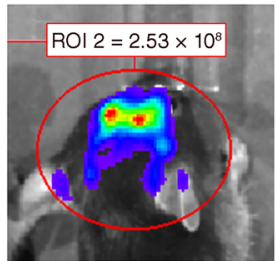

Endogenous glioma formation in vivo

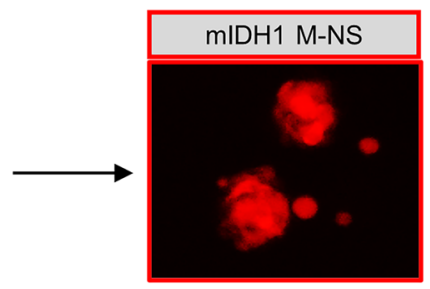

Culture mIDH1 and WT-IDH glioma derived neurospheres in vitro
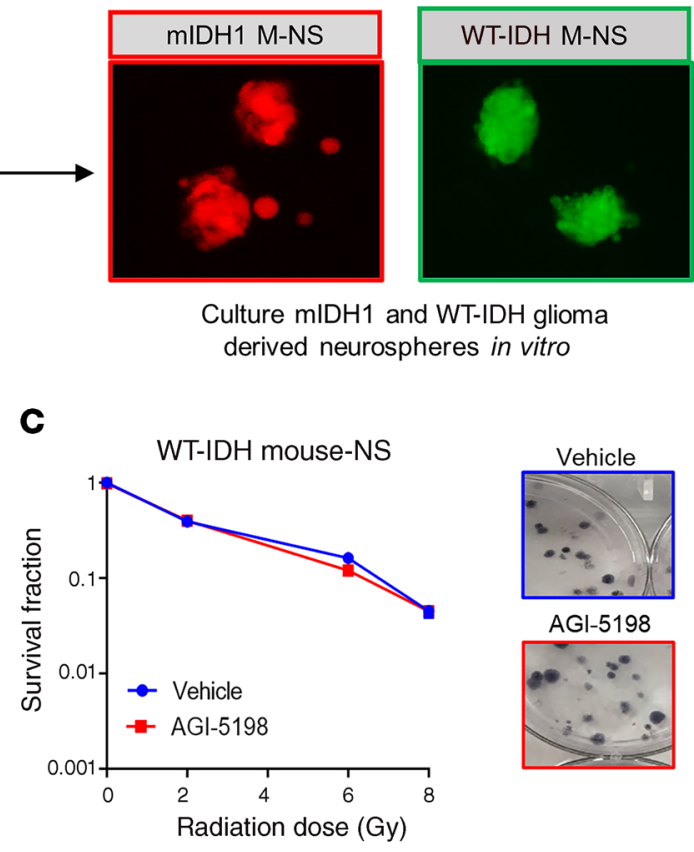

E
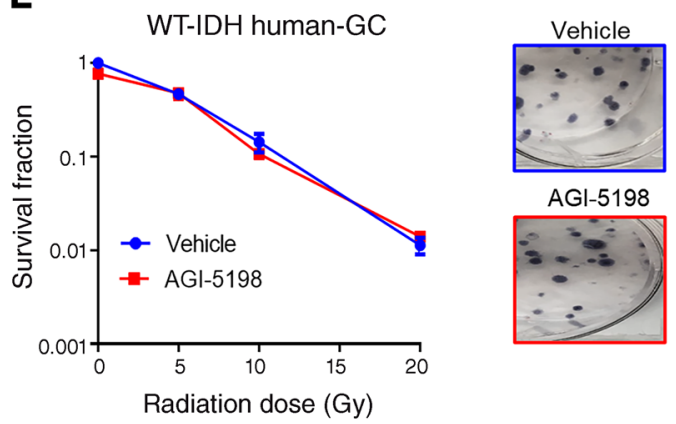

G Calreticulin (CRT)

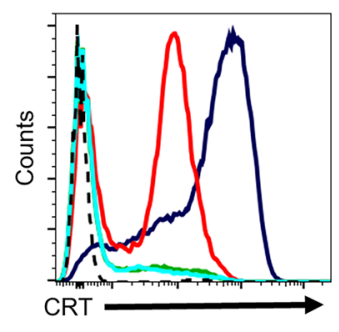

CRT: mIDH1 human-GC

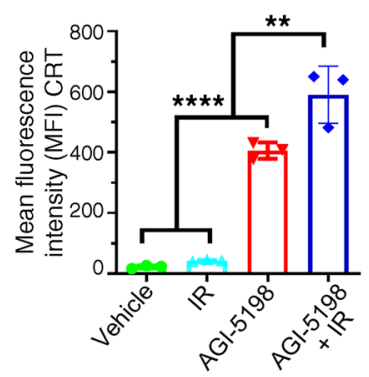

I

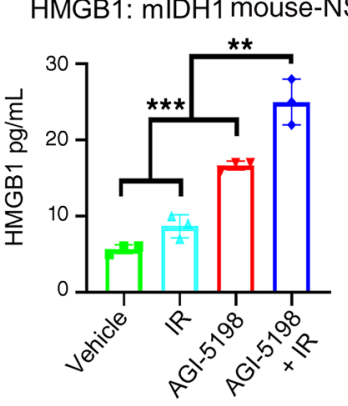

HMGB1: mIDH1 human-GC

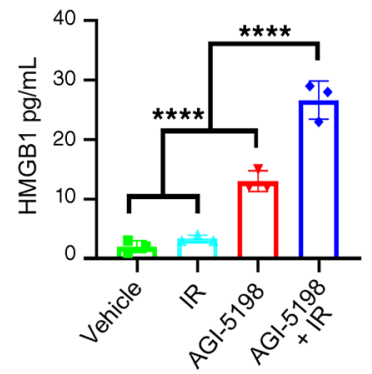

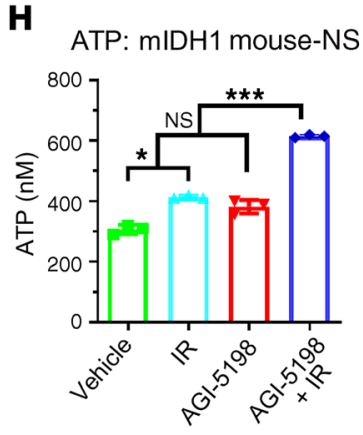

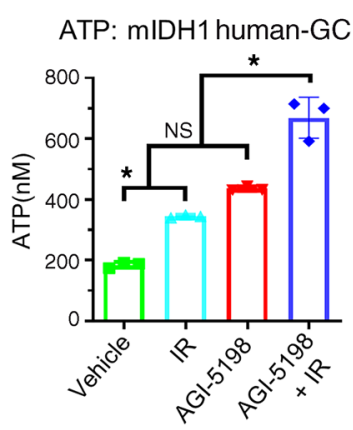


Figure 1. IDH1-R132H inhibition in mIDH1 mouse-NSs and humanGCs confers radiosensitivity and promotes release of DAMPs. (A) Mouse-NS cultures were generated from WT-IDH or mIDH1 glioma models. (B-E) Clonogenic assay of mIDH1 and WT-IDH mouse-NSs and human-GCs. (B and C) Mouse-NS were treated with 0-8 Gy ionizing radiation (IR) and $1.5 \mu \mathrm{M} \mathrm{AGI-5198} \mathrm{(red)} \mathrm{or} \mathrm{DMSO} \mathrm{vehicle} \mathrm{(blue).} \mathrm{(D} \mathrm{and}$ E) Human-GCs SJGBM2 (WT-IDH) and MGG119 (mIDH1) were treated with 0-20 Gy IR and $5 \mu \mathrm{M} \mathrm{AGI-5198} \mathrm{(red)} \mathrm{or} \mathrm{DMSO} \mathrm{vehicle} \mathrm{(blue).}{ }^{* * *} P<$ 0.0001 , nonlinear regression. Bars represent mean \pm SEM ( $n=3$ technical replicates). Representative images of colonies stained with crystal violet in each treatment group are shown under the survival fraction graphs. (F-I) Calreticulin (CRT), ATP, and HMGB1 expression levels within mIDH1 mouse-NSs and human-GCs. Mouse-NS were treated with 3 Gy IR and $1.5 \mu \mathrm{M}$ AGI-5198 for 72 hours. Human-GC were treated with $10 \mathrm{~Gy}$

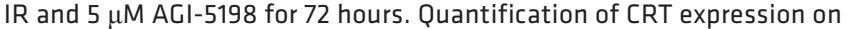
mIDH1 mouse-NSs and human-GCs after treatment is shown in $\mathbf{F}$ and $\mathbf{G}$, respectively. Representative histograms display CRT marker's expression levels (black, isotype control; green, DMSO vehicle; light blue, IR; red, AGI-5198; dark blue, AGI5198 + IR). ${ }^{* * *} P<0.0001$, ${ }^{* *} P<0.001$, 1 -way ANOVA. Bars represent mean \pm SEM ( $n=3$ technical replicates). (H) Quantification of ATP release in the supernatant of mIDH1 mouseNSs and human-GCs. ${ }^{*} P<0.01 ;{ }^{* *} P<0.01$; ${ }^{* *} P<0.001$, 1-way ANOVA. Bars represent mean \pm SEM ( $n=3$ technical replicates). (I) Quantification of HMCB1 release in the supernatant of mIDH1 mouse-NSs and human-GCs. ${ }^{* *} P<0.01 ;{ }^{* *} P<0.001 ;{ }^{* * *} P<0.0001$, 1-way ANOVA. Bars represent mean \pm SEM ( $n=3$ technical replicates).

We observed that the profile of myeloid-derived suppressor cells (MDSCs), macrophages, $\mathrm{CD}^{+} \mathrm{T}$ cells, and $\mathrm{CD} 4^{+} \mathrm{T}$ cells within the immune microenvironment of both mIDH1 glioma models were similar (Supplemental Figure 3). These data indicate that the immune microenvironment in SB-transposed gliomas and intracranial gliomas generated from SB-derived NS are comparable (Supplemental Figure 3).

Pharmacological inhibition of IDH1-R132H prolongs the survival of mIDH1 glioma-bearing mice. First, we sought to address if tumor-derived D-2HG production can be suppressed by IDH1R132H inhibitor AGI-5198 in vivo. Mice bearing either WT-IDH or mIDH1 glioma were treated with AGI-5198 or saline, as indicated in Figure 2A. Tumors were dissected from the brain and processed for UPLC-MS analysis 2 days after the last treatment dose (Supplemental Figure 4). Total D-2HG concentration was approximately 1.5 -fold $(0.29 \mu \mathrm{mol} / \mathrm{g})$ higher in mIDH1 tumor tissue compared with untreated normal brain tissue $(0.15$ $\mu \mathrm{mol} / \mathrm{g}$; Figure $2 \mathrm{~B})$. The levels of $\mathrm{D}-2 \mathrm{HG}$ in mIDH1 brain tumor tissue were reduced by approximately 2.4 fold $(P \leq 0.0001$; $0.12 \mu \mathrm{mol} / \mathrm{g}$ ) after treatment with AGI-5198 (Figure 2B). Furthermore, D-2HG levels in the mIDH1 brain tumor tissue of mice treated with AGI-5198 were similar to those observed in WT-IDH brain tissue $(0.15 \mu \mathrm{mol} / \mathrm{g}$; Figure $2 \mathrm{~B})$. These data demonstrate that AGI-5198 is capable of inhibiting IDH-R132H activity in the brain of our mouse glioma model.

Next, we characterized the in vivo cell cycle profile of mIDH1 tumor-bearing mice treated with AGI-5198, IR, or AGI-5198+IR. The frequency of mIDH1 cells undergoing mitotic G2/M phase $\left(\mathrm{pH} 3 \mathrm{Ser} 10^{+}\right)$of the cell cycle was approximately 1.5 -fold $(P \leq$ $0.001)$ lower in the AGI-5198 treatment group compared with the saline control and IR treatment groups (Figure 2C). In mice treated with AGI-5198+IR, we observed a 1.6-fold ( $P \leq 0.05$; Figure 2C) decrease in the frequency of $\mathrm{mIDH} 1$ cells undergoing mitosis compared with the AGI-5198 alone treatment group. Actively proliferating mIDH1 cells in S phase $\left(\mathrm{EdU}^{+}\right)$of the cell cycle were approximately 2.3 -fold $(P \leq 0.001)$ lower in the AGI-5198 treatment group compared with the saline and IR treatment groups (Figure 2C). Mutant IDH1 glioma cell proliferation was further reduced by approximately 6 -fold $(P \leq 0.001)$ in mice treated with AGI-5198+IR (Figure 2C)

Next, we asked whether AGI-5198 alone or AGI-5198+IR would improve MS and long-term survival of mIDH1 gliomabearing mice. Mutant IDH1 glioma-bearing mice were treated with (a) saline, (b) IR, (c) AGI-5198, or (d) AGI-5198+IR at the indicated dose and treatment schedule (Figure 2D). We monitored tumor growth using in vivo bioluminescence imaging. A significant decrease in bioluminescence signal was observed over time in mice treated with AGI-5198 or AGI-5198+IR compared with mice treated with saline or IR (Supplemental Figure $5)$. We observed a 1.3 -fold $(P \leq 0.001)$ increase in MS of mice in the AGI-5198-treated group (MS: $43 \mathrm{dpi}$ ), and a 1.5-fold ( $P \leq$ 0.001) increase in MS of mice in the AGI-5198+IR-treated group (MS: $50 \mathrm{dpi}$ ), when compared with the control mice in the saline treatment group (MS: $34 \mathrm{dpi}$ ) or IR treatment group (MS: $35 \mathrm{dpi}$ ). Treating mIDH1 glioma-bearing mice with PEG-400/ethanol vehicle control following the same treatment schedule as AGI5198 (Supplemental Figure 6A) did not confer a survival benefit (Supplemental Figure 6B).

Strikingly, we observed that $40 \%$ of the mIDH1 glioma-bearing mice treated with AGI-5198 or AGI-5198+IR survived long term (> $90 \mathrm{dpi}$ ) and remained tumor free (Figure 2E and Supplemental Table 1). Long-term survivors from the AGI-5198 alone or AGI-5198+IR treatment groups were rechallenged with mIDH1 mouse-NSs in the contralateral hemisphere. These animals remained tumor free without further treatment, whereas control mice implanted with glioma cells succumbed due to tumor burden (MS: 32 days; $P \leq 0.0001$ ) (Figure $2 \mathrm{~F}$, and Supplemental Figure 7). When sacrificed at $60 \mathrm{dpi}$ after tumor rechallenge, these mice showed no evidence of microscopic intracranial tumor (Supplemental Figure 7). These results suggest the development of anti-glioma immunological memory in mIDH1 glioma rechallenged animals previously treated with AGI-5198 or AGI-5198+IR.

We also assessed the therapeutic efficacy of AGI-5198 treatment in a WT-IDH glioma model. Mice bearing WT-IDH glioma were treated with either (a) saline, (b) IR, (c) AGI-5198, or (d) AGI5198+IR at the indicated doses and treatment schedule (Supplemental Figure 8A). We observed a 2.2-fold $(P \leq 0.01)$ increase in MS of WT-IDH glioma mice in the IR (MS: 54 dpi) and AGI5198+IR treatment groups (MS: $51 \mathrm{dpi}$ ) when compared with control mice in the saline (MS: $26 \mathrm{dpi}$ ) or AGI-5198 alone (MS: $24 \mathrm{dpi}$ ) treatment groups. These data demonstrate that AGI-5198 does not provide a survival benefit in WT-IDH glioma-bearing mice (Supplemental Figure 8).

Pharmacological inhibition of IDH1-R132H increases PD-L1 expression on mIDH1 glioma cells in vivo. Numerous studies have shown that PD-L1 is highly expressed by WT-IDH gliomas compared with mIDH1 gliomas (24). We evaluated the PD-L1 expression levels on mIDH1 and WT-IDH mouse glioma and immune cells in vivo. Mice were either implanted with WT-IDH or mIDH1 glioma cells and tumors were processed for flow cytometry anal- 
A

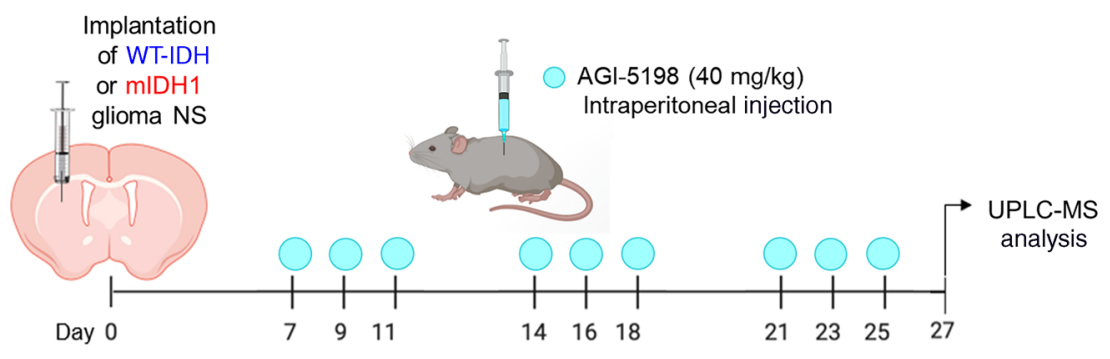

B

$\mathrm{D}-2 \mathrm{HG}$ measurement in the brain

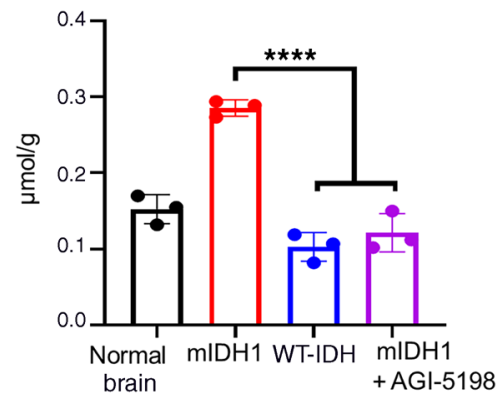

C
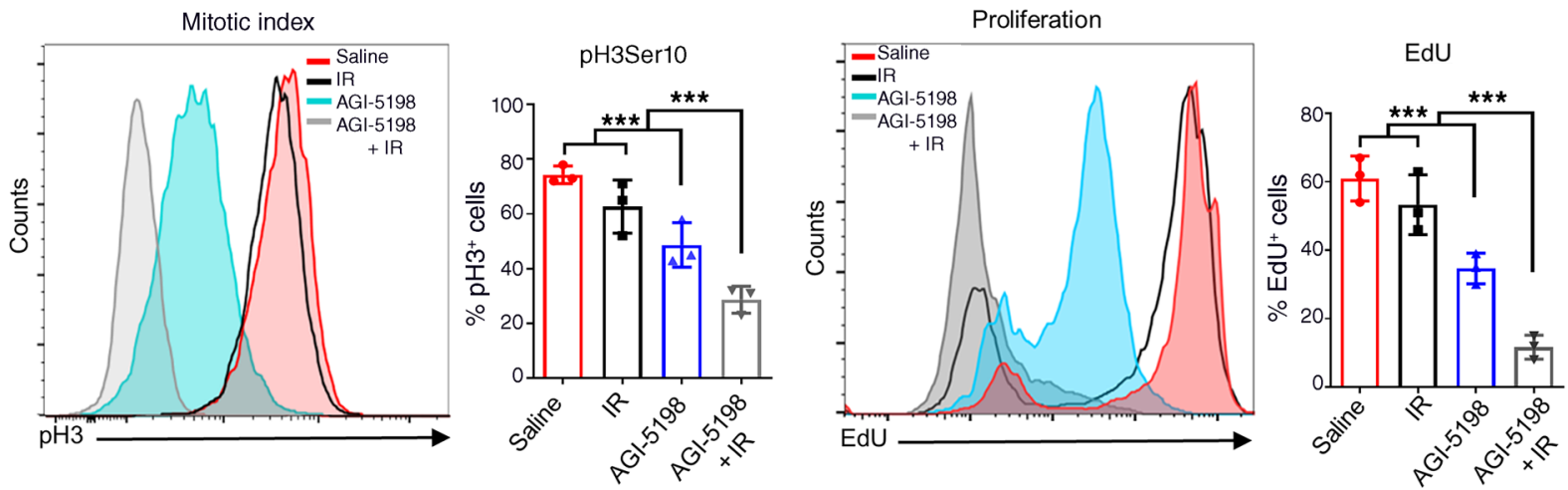

D

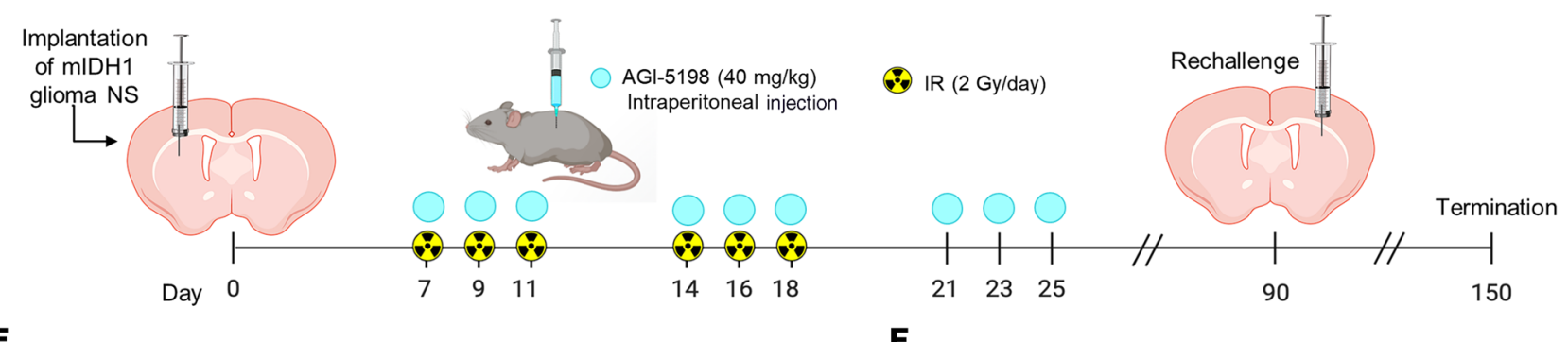

E

$\mathbf{F}$
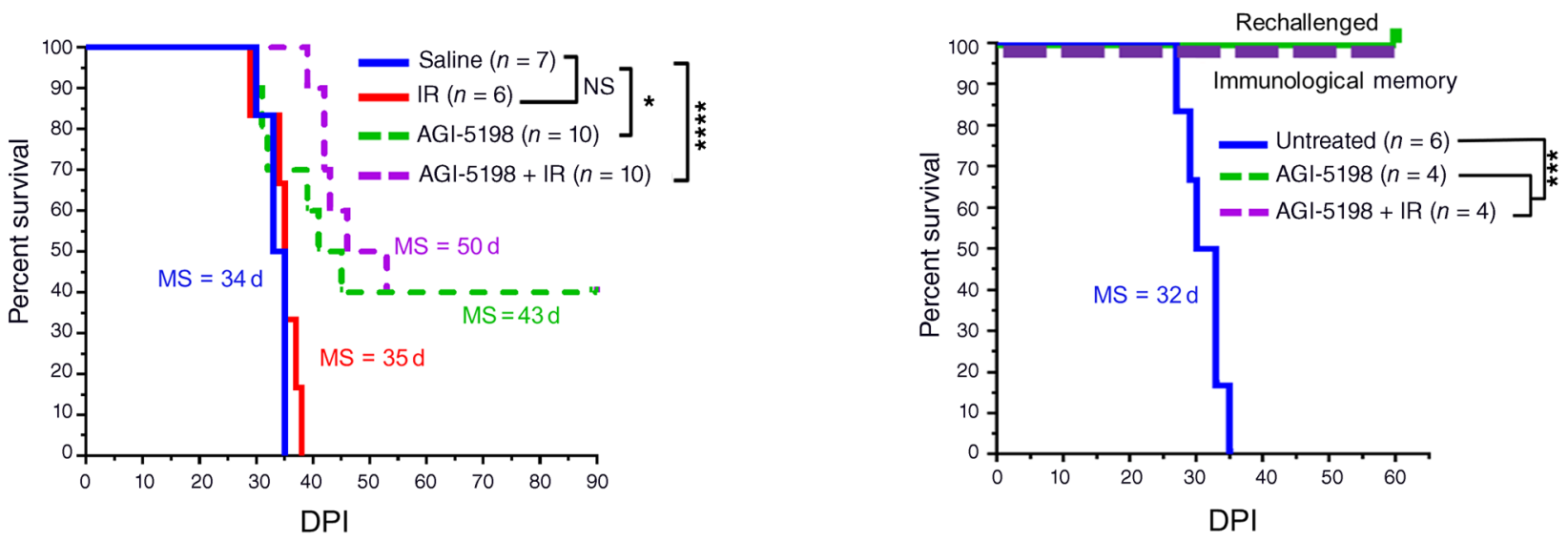
Figure 2. Inhibition of IDH1-R132H decreases the production of D-2HG in vivo, improves the survival of mIDH1 glioma-bearing mice, and elicits immunological memory. (A) Experimental design to assess D-2HC concentration in the brain TME. At 27 dpi brains were harvested for UPLC-MS analysis of D-2HG in the TME of normal mice (black), WT-IDH glioma-bearing mice (blue), mIDH1 glioma-bearing mice (red), and mIDH1 glioma-bearing mice treated with AGI-5198 (purple). (B) Quantification of $\mathrm{D}-2 \mathrm{HG}$ concentration in normal brain, mIDH1 tumor-bearing brain, WT-IDH tumor-bearing brain, and mIDH1 tumor-bearing brain plus ACI5198 treatment. ${ }^{* * *} P<0.0001,2$-way ANOVA. Bars represent mean \pm SEM ( $n=3$ biological replicates). (C) AGI-5198+IR treatment inhibits mIDH1 glioma cell proliferation in vivo. Mitotic cell division (mitotic index) in mIDH1 glioma was assessed by the phosphorylation status of Ser10 in Histone 3 ( $\mathrm{pH} 3$ ). Proliferation (S phase) of mIDH1 glioma was assessed by measuring the amount of EdU incorporation. Representative histograms display pH3- or EdU-positive cells (red, saline; black, IR; light blue, AGI-5198; gray, AGI-5198 + IR). *** $<0.001$, 1-way ANOVA. Bars represent mean \pm SEM ( $n=3$ biological replicates). (D) Experimental design to assess the survival of mIDH1 glioma-bearing mice treated with AGI-5198 therapy in combination with radiation. (E) Kaplan-Meier analysis of mice treated with saline $(n=7)$, IR $(n=6)$, AGI-5198 $(n=10)$, or AGI-5198+IR $(n=10)$. (F) Kaplan-Meier plot for rechallenged long-term survivors from (D) AGI-5198 $(n=4)$ or AGI-5198+IR $(n=4)$, and untreated animals $(n=6)$. Data were analyzed using the log-rank (Mantel-Cox) test. ${ }^{*} P<0.05$; ${ }^{* *} P<0.001 ;{ }^{* * *} P<0.0001$

ysis, as indicated in Figure 3A. We observed a 5.5-fold $(P \leq 0.001)$ decrease in PD-L1 expression on CD45 / Nestin ${ }^{+}$mIDH1 glioma cells when compared with WT-IDH glioma cells (Figure 3B). There was also a 2.3-fold $(P \leq 0.01)$ decrease in PD-L1 expression on $\mathrm{CD} 45^{+}$immune cells in the mIDH1 glioma TME when compared with WT-IDH glioma TME (Figure 3C). Next, we evaluated whether AGI-5198 would change the PD-L1 expression on MIDH1 glioma cells or immune cells in vivo. Mice bearing mIDH1 tumors were treated with AGI-5198, as indicated in Figure 3A. Administration of AGI-5198 to mIDH1 glioma-bearing mice led to a 3 -fold ( $P \leq 0.001)$ increase in the PD-L1 expression on the CD45 ${ }^{-} /$ Nestin $^{+}$tumor cells compared with untreated mIDH1 gliomabearing mice (Figure 3B). There was no difference in PD-L1 expression on $\mathrm{CD}_{4} 5^{+}$immune cells in the mIDH1 glioma TME treated with AGI-5198 when compared with the untreated mIDH1 glioma TME (Figure 3C). Using immunohistochemistry, we confirmed the expression of PD-L1 on Nestin ${ }^{+}$cells in brain sections from WT-IDH, mIDH1, or mIDH1 glioma-bearing mice treated with AGI5198. PD-L1 expression was markedly decreased in mIDH1 glioma compared with WT-IDH glioma (Figure 3D). PD-L1 expression in $\mathrm{mIDH} 1$ glioma treated with AGI-5198 increased to levels comparable to WT-IDH glioma (Figure 3D).

Since mIDH1 induces a global hypermethylation phenotype, we next evaluated the effect of AGI-5198 treatment on the DNA methylation status within $\mathrm{CpG}$ islands of the CD274 promoter in WT-IDH and mIDH1 mouse-NSs (Supplemental Figure 9). We found that CD274 methylation levels were approximately 9-fold ( $\mathrm{P}<0.01)$ higher in $\mathrm{mIDH} 1$ mouse-NSs treated with vehicle control compared with WT-IDH mouse-NSs (Figure 3E; Supplemental Figure 10). The mIDH1 mouse-NSs treated with AGI5198 displayed approximately 9 -fold $(\mathrm{P} \leq 0.01)$ lower PD-L1 DNA methylation levels relative to vehicle control (Figure 3E, and Supplemental Figure 10). There were no differences in DNA methylation levels for WT-IDH mouse-NSs treated with AGI-
5198 compared with vehicle control (Figure 3E, and Supplemental Figure 10). Taken together, these results demonstrate that IDH1-R132H inhibition specifically impacts the PD-L1 expression on mIDH1 glioma cells.

Consistent with our results, TCGA analysis of grades II and III glioma patients harboring IDH1-R132H with TP53 and ATRX inactivating mutations revealed approximately 1.1 -fold $(P \leq 0.001)$ lower expression of PD-L1 (gene annotation denoted as CD274) (25) mRNA compared with WT-IDH glioma patients (Figure 4A). We also observed that patients with grades II and III glioma harboring IDH1-R132H with 1p/19q codeletion have approximately 1.1-fold $(P \leq 0.001)$ lower expression of CD274 mRNA compared with patients with WT-IDH glioma (Figure 4A).

Since D-2HG inhibits DNA and histone demethylases (13), altering the epigenome and gene expression patterns, the change in PD-L1 expression was mainly observed in mIDH1 glioma samples (Figure 3, A-E). Therefore, we asked if there were differences in the CD274 promoter methylation status between the patient WT-IDH and mIDH1 tumors. We analyzed the promoter methylation profiles of the CD274 gene in grades II and III glioma samples from the TCGA. We classified the samples into 3 groups: mIDH1 with 1p/19q codeletion (mIDH1-Codel), mIDH1 with non 1p/19q codeletion (mIDH1-nonCodel), and WT-IDH group. Within the CpG island of the CD274 gene, we identified a total of 5 probes (Cg15837913, Cg19724470, Cg14305799, Cg13474877, Cg02823866). Using 3 of the 5 probes (Cg15837913, Cg19724470, and Cg14305799), we found that DNA methylation was significantly higher in both mIDH1-Codel and mIDH1nonCodel as compared with the WT-IDH group (Figure 4B). The presence of DNA hypermethylation within the CD274 promoter region in the mIDH1 samples is correlated with the lower CD274 gene expression, suggesting the involvement of epigenetic regulation of CD274 gene expression in the mIDH1 glioma. Since IDH1$\mathrm{R} 123 \mathrm{H}$ is also encountered in other cancers, we assessed the CD274 methylation profile in AML. We did not find differences in DNA methylation in CpG sites within the CD274 promoter of AML patient samples (Supplemental Figure 11). These data indicate that CD274 expression is also dependent on other genetic lesions coexpressed with mIDH1 within the tumor. In the glioma subtypes we evaluated, IDH-R132H is expressed in the context of TP53 and ATRX mutations or 1p19q codeletion, differing from AML, where ATRX inactivating mutations or $1 \mathrm{p} 19 \mathrm{q}$ codeletion are not present. These data indicate that IDH-R132H is directly involved in regulating CD274 expression in grade II and III gliomas. In addition, treating human mIDH1 cells (SF10602), which endogenously express IDH1-R132H, ATRX, and TP53 inactivating mutations, with AGI-5198 lowered the CD274 DNA methylation levels relative to the vehicle control (Figure $4 \mathrm{C}$, and Supplemental Figure 12). Taken together, these results demonstrate that IDH1-R132H inhibition specifically impacts the regulation of PD-L1 expression in MIDH1 gliomas.

Therapeutic efficacy of IDH1-R132H inhibition, SOC, and anti$P D-L 1$ blockade in an intracranial mIDH1 glioma model. Given that PD-L1 expression increased on mIDH1 glioma cells when treated with AGI-5198 (Figure 3B) and that MGMT methylation status is high in these cells (Supplemental Figure 13), we investigated whether combining AGI-5198 therapy with SOC (TMZ+IR) and 
A

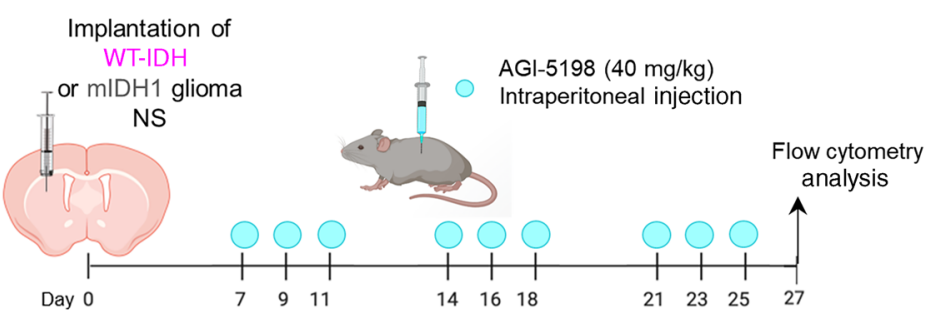

C
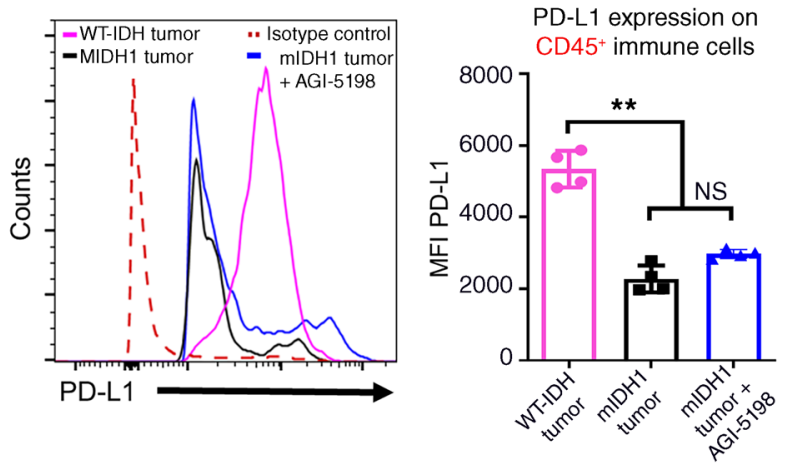

B

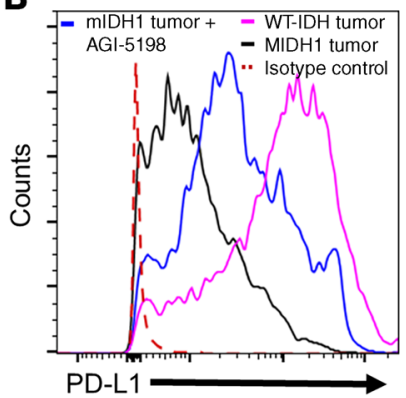

PD-L1 expression on CD45-/Nestin+ tumor cells

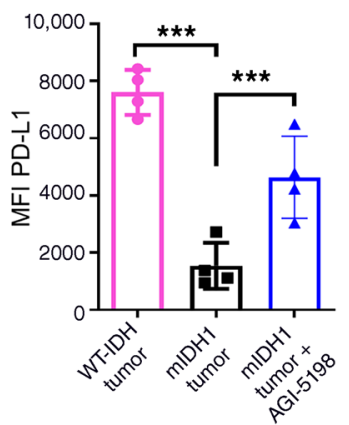

D
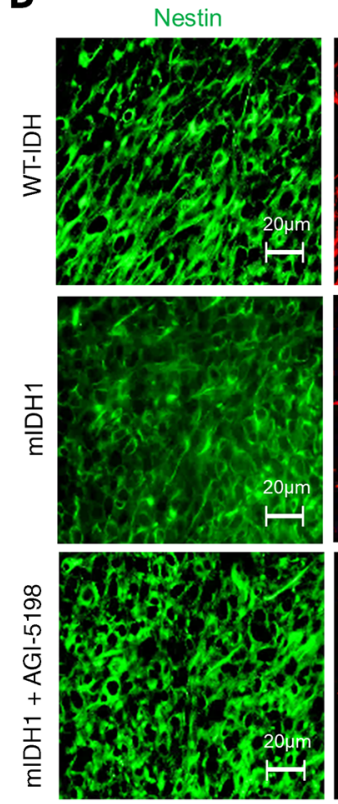

PD-L1
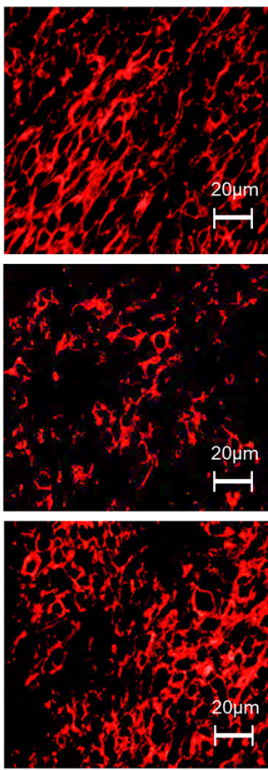

DAPI
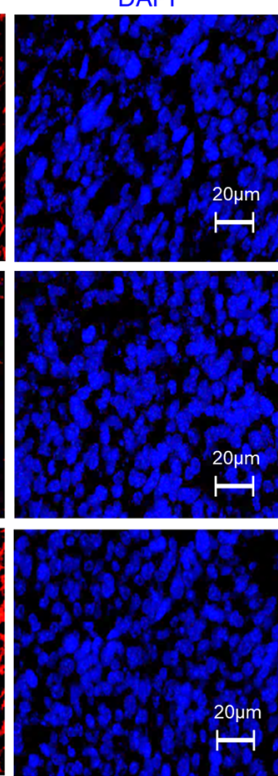

E
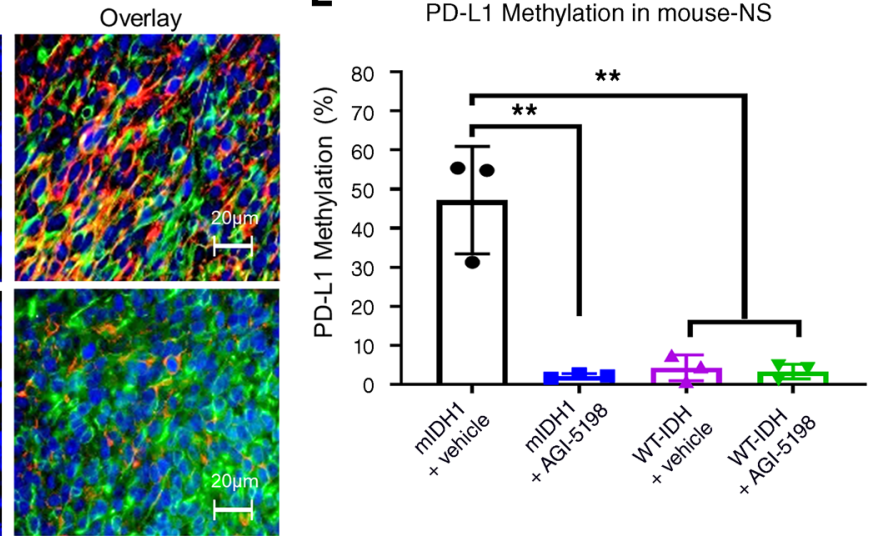

Figure 3. Inhibition of IDH1-R132H increases PD-L1 expression levels on mouse mIDH1 glioma cells. (A) Experimental design to characterize PD-L1 expression on glioma cells and immune cells. (B) Representative histograms displaying PD-L1 expression levels on CD45-/Nestin ${ }^{+} / \mathrm{Katushka}^{+}$glioma cells or (C) on CD45+ immune cells in the TME (purple, WT-IDH tumor; black, mIDH1 tumor; blue, mIDH1 tumor treated with AGI-5198). ${ }^{* *} P<0.01$; ${ }^{* * *} P<0.001 ; 1$-way ANOVA. Bars represent mean \pm SEM ( $n=4$ biological replicates). (D) Immunofluorescence staining of Nestin (green), PD-L1 (red), and DAPI (blue) in $5 \mu \mathrm{m}$ brain tumor sections from WT-IDH ( $24 \mathrm{dpi}$ ), mIDH1 (35 dpi), or mIDH1+AGI-5198 (43 dpi) animals. (E) Quantification of PD-L1

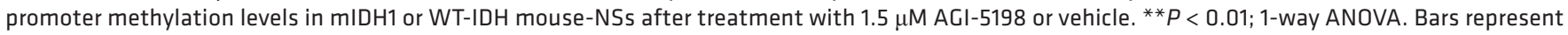
mean $\pm \operatorname{SEM}(n=3$ technical replicates).

anti-PD-L1 ( $\alpha$ PD-L1) immune checkpoint blockade would provide therapeutic benefit to mIDH1 glioma-bearing mice. Mice bearing mIDH1 tumors were treated at the indicated doses and treatment schedule (Figure 5A). Administration of TMZ alone (MS: 39 dpi), SOC (TMZ+IR) alone (MS: 38 dpi), or SOC $+\alpha$ PD-L1 (MS: 39 dpi) to mice bearing mIDH1 tumors conferred a 1.1 -fold $(P<0.01)$ survival benefit compared with saline (MS: 35 dpi; Figure 5B). Administration of $\alpha$ PD-L1 (MS:43 dpi), $\alpha$ PDL+TMZ (MS: $42 \mathrm{dpi}$ ), or $\alpha$ PD-L1+ IR (MS: 43 dpi) also conferred a modest approximately 1.2-fold $(P<0.01)$ survival benefit (Figure $5 \mathrm{~B})$.

Treating mIDH1 tumor-bearing mice with AGI-5198+SOC (MS: 52 dpi) enhanced the efficacy of SOC alone (MS: $38 \mathrm{dpi}$ ), 
A

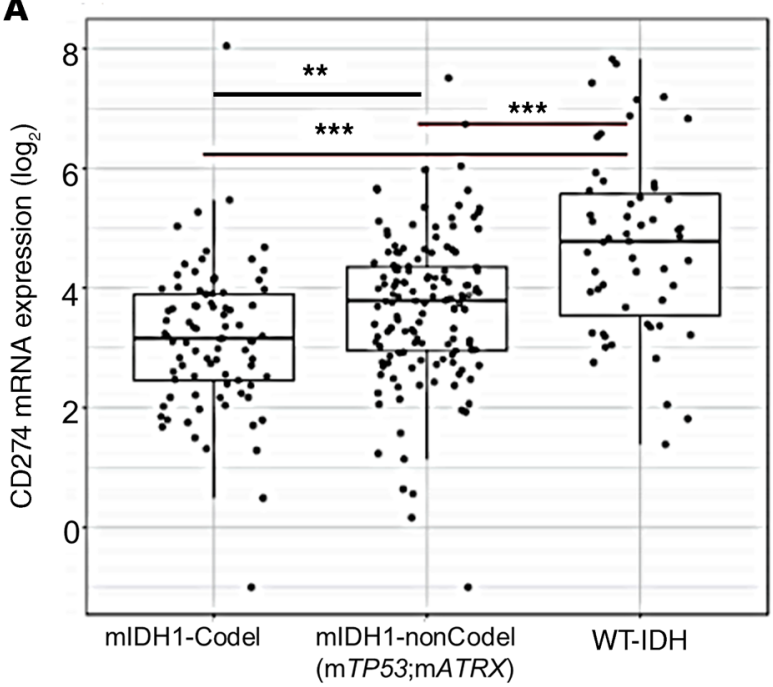

C

CD274 Methylation in mIDH1 human-GC

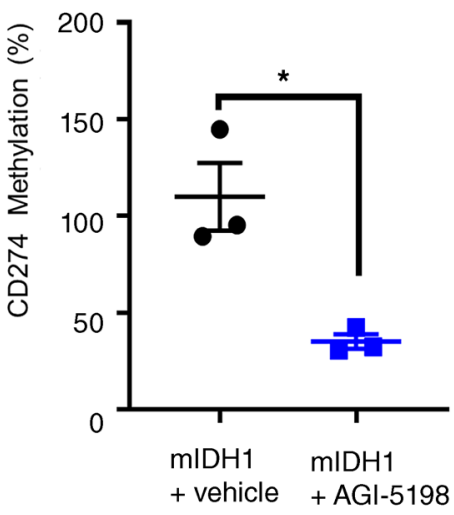

B

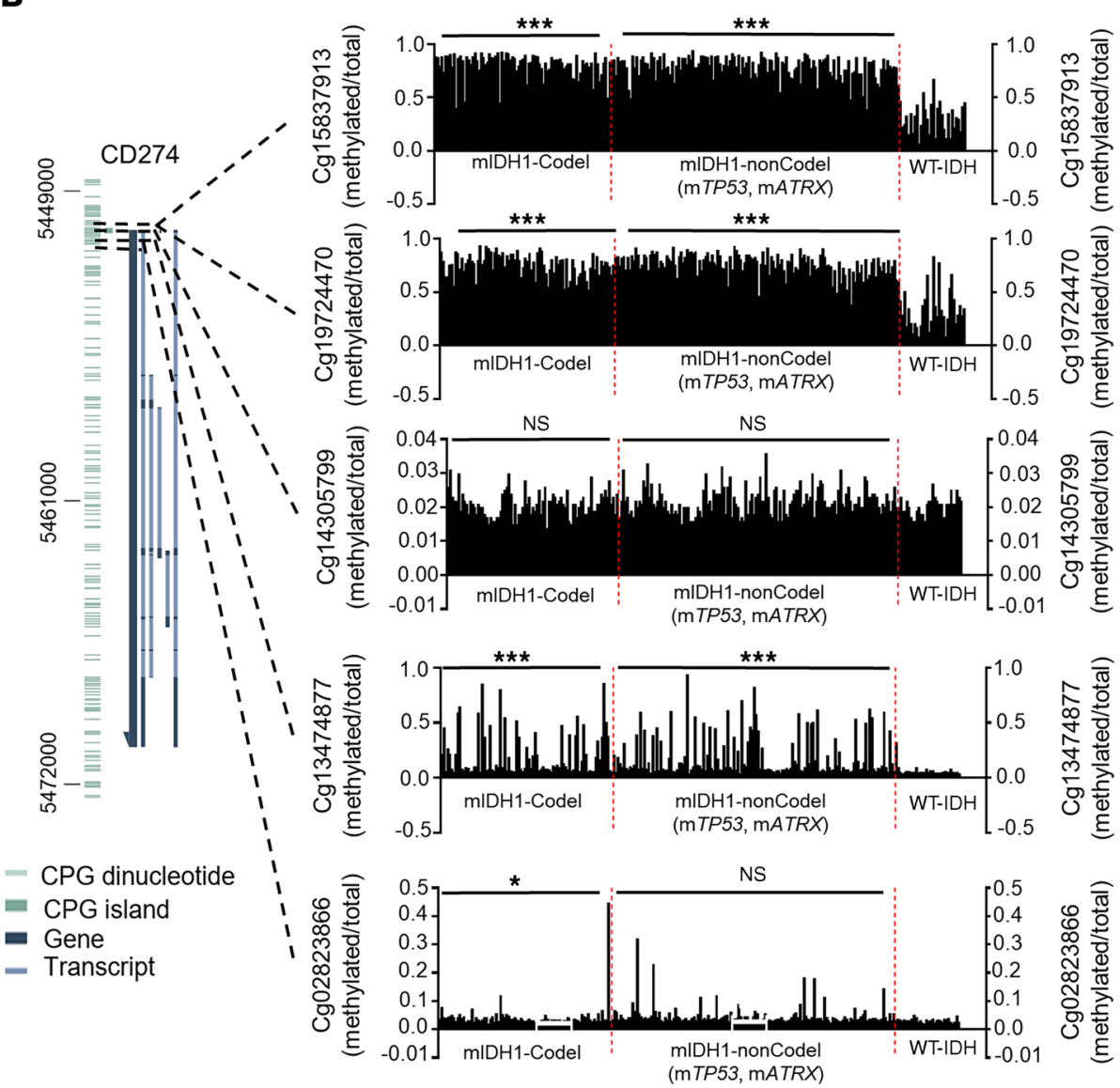

Figure 4. Regulation of PD-L1 expression in human mIDH1 glioma cells. (A) Analysis of CD274 (PD-L1) gene expression for IDHmut-codel $(n=85)$, IDH1mut-noncodel ( $n=141)$, and WT-IDH $(n=55)$ grade II and III glioma patients. RNAseq data were obtained from TCCA (GlioVis platform). Graph displays the $\log _{2}$ expression value of CD274 mRNA expression: each dot represents 1 patient. ${ }^{* *} P<0.001$, Tukey's Honest Significant Difference test. (B) DNA methylation levels within cg15837913, cg19724470, cg14305799, cg13474877, or cg02823866 probes in the $\mathrm{CpG}$ island of CD274 promoter were determined for IDH mut-codel ( $n=$ 85), IDH1mut-noncodel ( $n=$ $141)$, and WT-IDH ( $n=55)$ grade II and III glioma patients. Each black bar represents 1 patient. DNA methylation status was assessed for TCGA data using MEXPRESS. ${ }^{*} P<0.05$; ${ }^{* * *} P<0.001$; Tukey's Honest Significant Difference test. (C) Quantification of PD-L1 promoter methylation levels in mIDH1 human cells after treatment with $5 \mu \mathrm{M} \mathrm{AGI-5198.}$ ${ }^{*} P<0.05$; unpaired, 2 -tailed Student $t$ test. Bars represent mean \pm SEM ( $n=3$ technical replicates). and resulted in 20\% long-term survivors (Figure 5C). We also observed that the addition of AGI-5198 to TMZ $+\alpha$ PD-L1 (MS: $42 \mathrm{dpi}$ ) or IR $+\alpha$ PD-L1 (MS: $61 \mathrm{dpi}$ ) treatments significantly enhanced therapeutic efficacy in mIDH1 glioma-bearing mice (Figure 5C). Notably, in the AGI-5198+TMZ+ $\alpha$ PD-L1 and AGI-5198 $+\mathrm{IR}+\alpha \mathrm{PD}-\mathrm{L} 1$ treatment groups, $40 \%$ of mice were long-term survivors, but the highest survival advantage was observed in the AGI-5198+SOC+ $\alpha$ PD-L1 treatment group
(MS: not reached), in which $60 \%$ of mice were long-term survivors (Figure 5C, and Supplemental Tables 2-4). Our statistical analyses demonstrated that the addition of $\alpha$ PD-L1 to AGI$5198+$ SOC can improve the 90 -day survival rate by $40 \%(95 \%$ CI: $0 \%-95 \%, 1$-sided $P=0.08)$. The estimated hazard ratio is 0.3 (95\% CI: $0.06-1.7, P=0.2$ ), indicating that $\alpha$ PD-L1 can reduce the hazard of death by $70 \%$. Our survival data did not reach statistical significance due to the study's small sample 
A

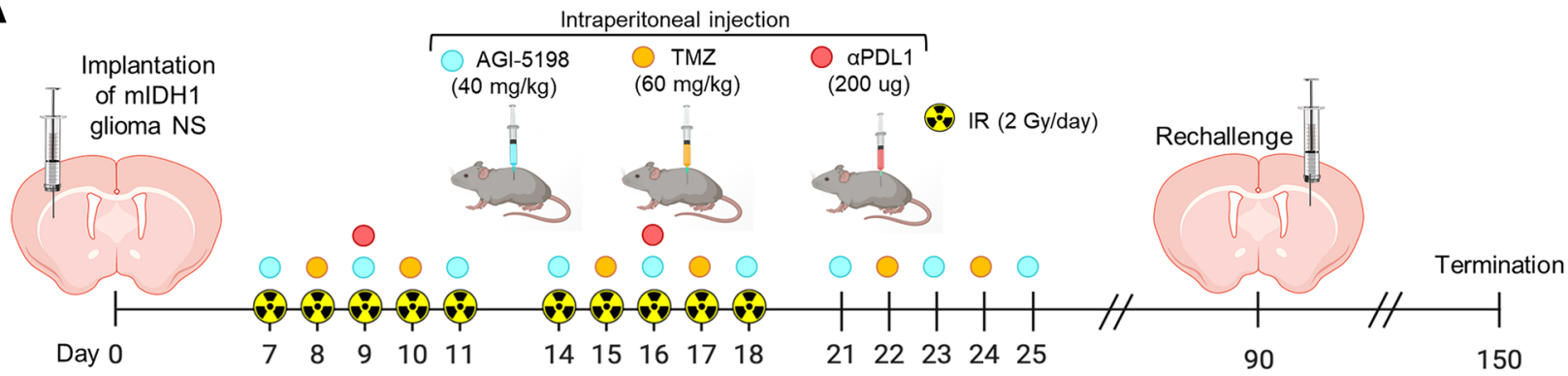

B

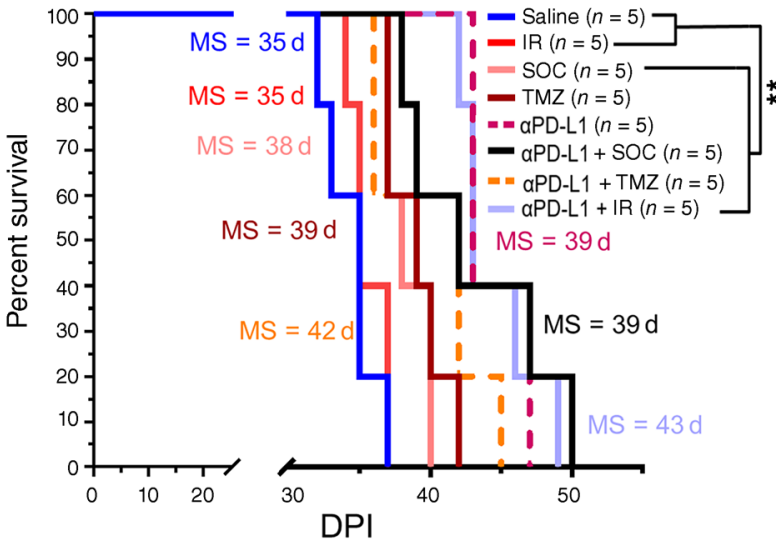

C

D

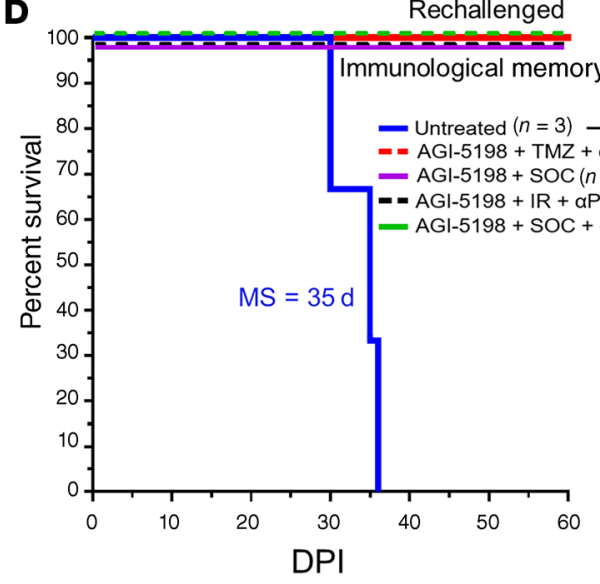

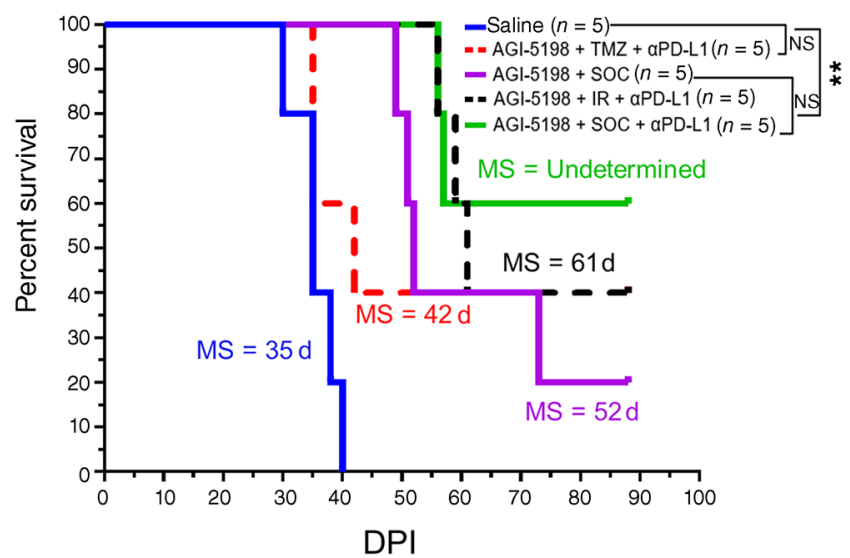

E

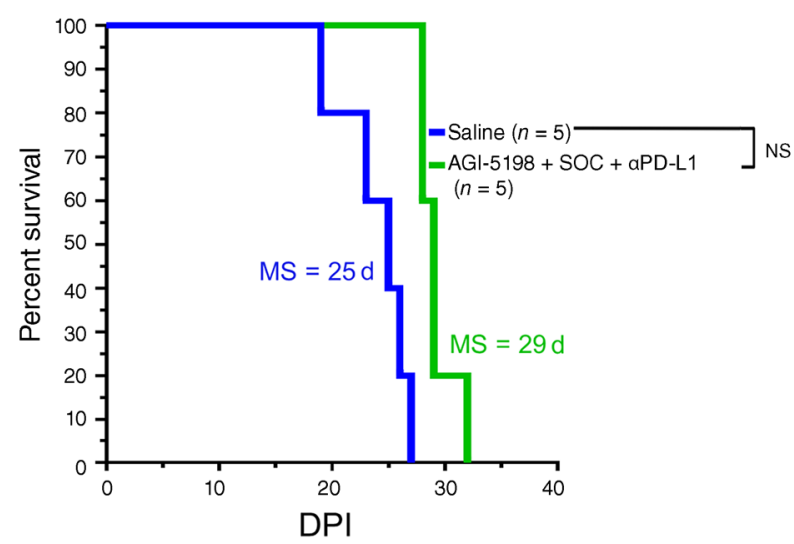

$\mathbf{F}$
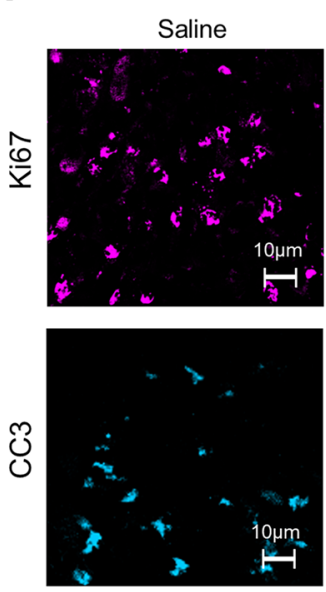

AGI-5198 + SOC
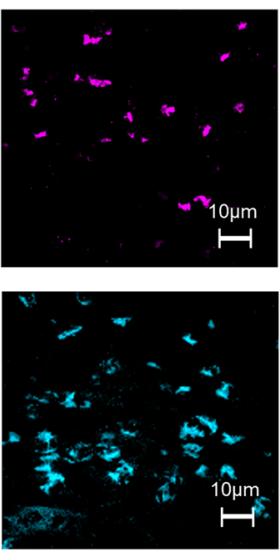
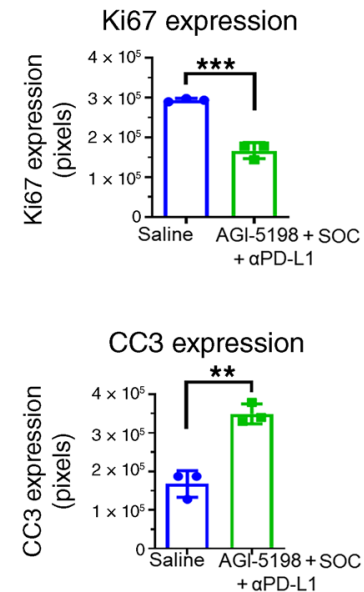

G

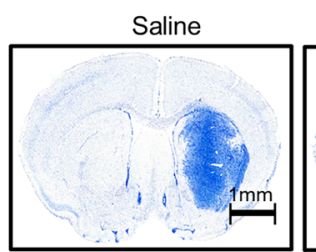

AGI-5198+SOC $+\alpha \mathrm{PD}-\mathrm{L} 1$

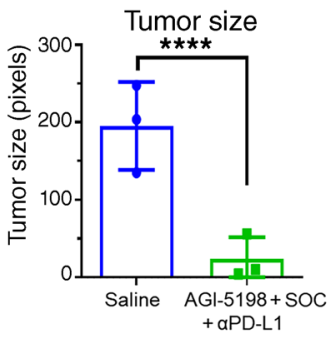


Figure 5. Inhibition of IDH1-R132H in combination with SOC and $\alpha$ PD-L1 exhibits enhanced survival of mIDH1 glioma-bearing mice. (A) Experimental design to assess survival for AGI-5198+SOC $+\alpha \mathrm{PD}-\mathrm{L} 1$ therapy. (B) Kaplan-Meier survival analysis of C57BL/6 mice treated with saline $(n=5)$, SOC $(n=5)$, TMZ $(n=5), \alpha \mathrm{PD}-\mathrm{L} 1+\mathrm{SOC}(n=5), \alpha \mathrm{PD}-\mathrm{L} 1(n=5)$, $\alpha \mathrm{PD}-\mathrm{L} 1+\mathrm{TMZ}(n=5)$, and IR+aPDL $(n=5)$. (C) Kaplan-Meier survival analysis of C57BL/ 6 mice treated with saline $(n=5), A G I-5198+T M Z+\alpha P D-L 1$ $(n=5)$, AGI-5198+SOC $(n=5)$, AGI-5198+IR+ $\alpha$ PD-L1 $(n=5)$, and AGI$5198+S O C+\alpha P D-L 1(n=5)$. (D) Kaplan-Meier survival plot for rechallenged long-term survivors from (C) AGI-5198+TMZ+ $\alpha$ PD-L1 $(n=1)$, AGI-5198+SOC $(n=2)$, AGI-5198+IR+ $+\alpha$ PD-L1 $(n=2)$, and AGI-5198+SOC+ $\alpha$ PD-L1 $(n=3)$ groups, and control mice $(n=3)$. Data were analyzed using the log-rank (Mantel-Cox) test. ${ }^{* *} P<0.001 ;{ }^{* *} P<0.001$. (E) Kaplan-Meier analysis of mIDH1 glioma-bearing CD8-KO mice treated with saline $(n=5)$ or AGI$5198+S O C+\alpha P D-L 1(n=5)$. (G) C57BL/ 6 mice bearing mIDH1 glioma were treated with saline or AGI-5198+SOC+ $\alpha$ PD-L1 as detailed in A. At $27 \mathrm{dpi}$, brains were harvested for immunohistochemistry analysis. Immunofluorescence staining for Ki67 and CC3 was performed on $50 \mu \mathrm{m}$ vibratome tumor sections. Bar graphs represent total number of positive cells for Ki67 and CC3 in saline or ACI-5198+SOC+ $\alpha$ PD-L1 treatment groups. ${ }^{* *} P$ $<0.01 ;{ }^{* * *} P<0.001$, unpaired $t$ test. Bars represent mean \pm SEM ( $n$ $=3$ biological replicates). (F) Nissl staining of $50 \mu \mathrm{m}$ brain sections at $27 \mathrm{dpi}$ from mIDH1 tumor-bearing mice treated with saline and AGI$5198+$ SOC $+\alpha$ PD-L1. ${ }^{* * *} P<0.001$; unpaired $t$ test. Bars represent mean \pm $\operatorname{SEM}(n=3$ biological replicates).

size, but our results suggest that adding $\alpha \mathrm{PD}-\mathrm{L} 1$ could enhance the efficacy of AGI-5198+SOC.

The long-term survivors from the AGI-5198+TMZ $+\alpha$ PD-L1, AGI-5198+SOC, AGI-5198+IR $+\alpha$ PD-L1, or AGI$5198+$ SOC $+\alpha$ PD-L1 treatment groups were rechallenged with mIDH1 tumors in the contralateral hemisphere (Figure 5A). These animals remained tumor free without further treatment, compared with untreated control mice implanted with tumors, which succumbed due to tumor burden (MS: 35 days; $P \leq 0.0001$ ) (Figure 5D). When sacrificed at $60 \mathrm{dpi}$ after tumor rechallenge, mice showed no evidence of microscopic intracranial tumor (Supplemental Figures 14 and 15), suggesting the development of immunological memory.

To determine whether the efficacy of AGI-5198+SOC $+\alpha$ PD-L1 is mediated by the adaptive immune system, CD8-KO mice were implanted with $\mathrm{mIDH} 1$ cells and treated with saline or AGI-5198+SOC $+\alpha$ PD-L1 at the indicated dose and treatment schedule (Figure 5A). There was not a statistically significant difference in MS in CD8-KO mice treated with saline or AGI$5198+$ SOC $+\alpha$ PD-L1. These data indicate the critical role that $\mathrm{CD}^{+} \mathrm{T}$ cells have in mediating the observed therapeutic response induced by AGI-5198+SOC $+\alpha \mathrm{PD}-\mathrm{L} 1$ treatment (Figure $5 \mathrm{E}$ ).

Subsequently, we analyzed the in vivo expression of Ki67 (proliferation marker), cleaved caspase 3 (CC3; apoptosis marker), and CD8 (cytotoxic T cell marker) in mIDH1 tumors 2 days after saline, or AGI-5198+SOC+ $\alpha$ PD-L1 treatments (27 dpi, Figure 5A). Combining AGI-5198 treatment with SOC and $\alpha \mathrm{PD}-\mathrm{L} 1$ resulted in a significant decrease in Ki67 expression $(P<0.0001)$ and an increase in CC3 expression $(P<0.01)$ compared with the saline-treated group (Figure $5 \mathrm{~F}$ ). We also observed increased infiltration of $\mathrm{CD}^{+} \mathrm{T}$ cells $(P<0.01)$ in tumors treated with AGI$5198+$ SOC $+\alpha$ PD-L1 when compared with saline (Supplemental Figure 16). Additionally, we quantified the size of the tumor 2 days after AGI-5198+SOC+ $\alpha$ PD-L1 or saline treatment (27 dpi). We observed a 3-fold $(P<0.0001)$ decrease in tumor size in the mice treated with AGI-5198+SOC+ $\alpha$ PD-L1 (Figure 5G).

We also assessed complete blood cell counts (CBC), serum biochemistry for aminotransferase (ALT), bilirubin, urea (BUN) and creatinine for mice treated with saline or AGI$5198+$ SOC $+\alpha$ PD-L1 2 days following the last treatment dose (Figure 5A). No differences were observed in relation to $\mathrm{CBC}$ between the treatment groups (Supplemental Figure 17). The levels of ALT, BUN, and creatinine were within the normal range, indicating normal functioning liver and renal systems (Supplemental Table 5). The mice were examined daily by a board-certified veterinarian and no overt signs of respiratory distress, adverse neurological signs, or weight loss were observed. Furthermore, liver tissue sections from both treatment groups showed no signs of necrosis, inflammation, or changes in cellular structures (Supplemental Figure 18).

Long-term survivors from the AGI-5198+SOC+ $\alpha$ PD-L1 treatment group rechallenged with mIDH1 glioma and not receiving any additional treatment were euthanized 60 days later (Figure 5A). Brains were assessed by immunohistochemical staining for myelin basic protein (MBP) as a marker for myelin sheaths. We did not observe evidence of demyelination in mIDH1 glioma rechallenged mice from the AGI-5198+SOC $+\alpha$ PD-L1 treatment group compared with the saline-treated mice (Supplemental Figure 15). We also assessed GFAP expression in the brain tumor sections after AGI-5198+SOC $+\alpha$ PD-L1 treatment and compared it with the untreated mIDH1 glioma-bearing controls (Supplemental Figure 15). The rechallenged long-term survivors from the AGI$5198+$ SOC $+\alpha$ PD-L1 treatment group did not show any evidence of intracranial tumor mass in either hemisphere. We observed reactive astrocytes surrounding the remaining scar tissue at the site of implantation of the primary tumor (Supplemental Figure 15). Overall, our data demonstrate that administration of AGI-5198+SOC $+\alpha$ PD-L1 treatment confers an overall survival advantage and elicits an anti-mIDH1 glioma immune response with no overt acute or long-term off-target toxicity effects.

Expansion of $\mathrm{mIDH} 1$ anti-glioma-specific cytotoxic $T$ cells in response to IDH1-R132H inhibition in combination with SOC and $\alpha P D-L 1$ immune checkpoint blockade. We next aimed to study the anti-mIDH1 glioma-specific immune response elicited by AGI$5198+$ SOC $+\alpha$ PD-L1 therapy. Mice bearing mIDH1 tumors harboring a surrogate tumor antigen, ovalbumin (OVA), were treated with saline, AGI-5198+SOC, or AGI-5198+SOC+ $\alpha$ PD-L1, as indicated in Figure 6A. Mice were euthanized 2 days after the last treatment dose and brains were processed for flow cytometry analysis to characterize the immune cell infiltration in the TME. Treatment of mIDH1 glioma-bearing mice with AGI-5198+SOC therapy induced a 1.6-fold $(P \leq 0.05)$ increase in the percentage of tumor-infiltrating plasmacytoid DCs (pDCs: CD $45^{+} / \mathrm{CD} 11 \mathrm{c}^{+} /$ $\left.\mathrm{B} 220^{+}\right)$and a 2.2 -fold $(P \leq 0.001)$ increase in conventional DCs (cDCs: CD 45 ${ }^{+} / \mathrm{CD}_{11 \mathrm{c}^{+}} / \mathrm{B} 220^{-}$) compared with saline-treated mice (Figure 6B). In mice treated with AGI-5198+SOC+ $\alpha$ PD-L1, we observed an additional 1.5-fold ( $P \leq 0.05$; Figure 6B) increase in tumor-infiltrating pDCs and approximately 1.3-fold ( $\mathrm{P} \leq 0.001$; Figure $6 \mathrm{~B}$ ) increase in tumor-infiltrating $\mathrm{cDCs}$ compared with mice treated with AGI-5198+SOC. 
A

TMZ $\bigcirc$ aPDL1

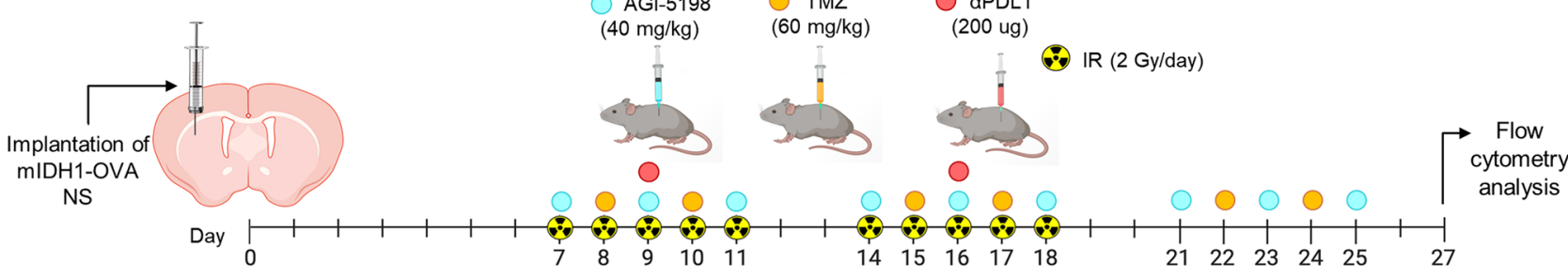

B
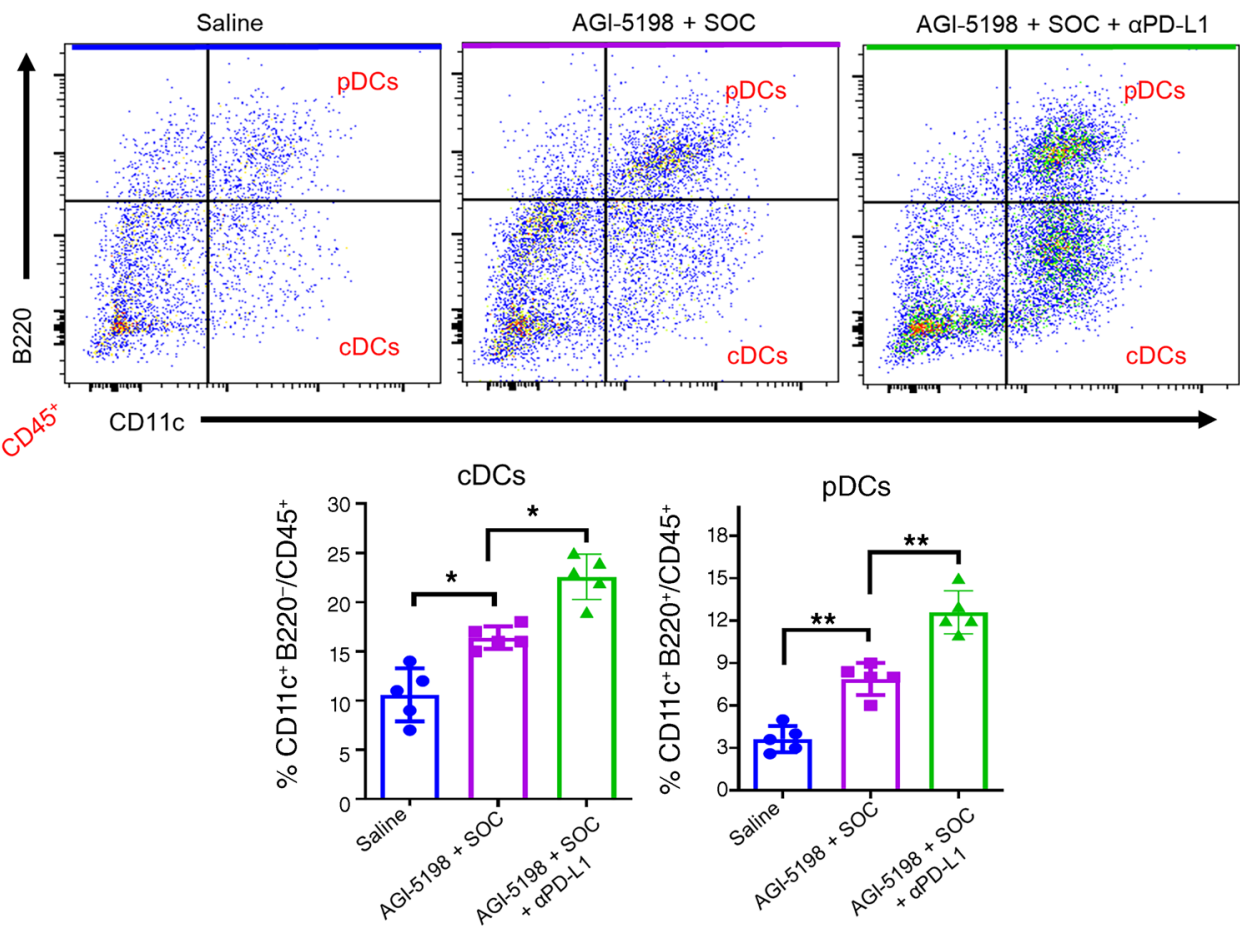

C

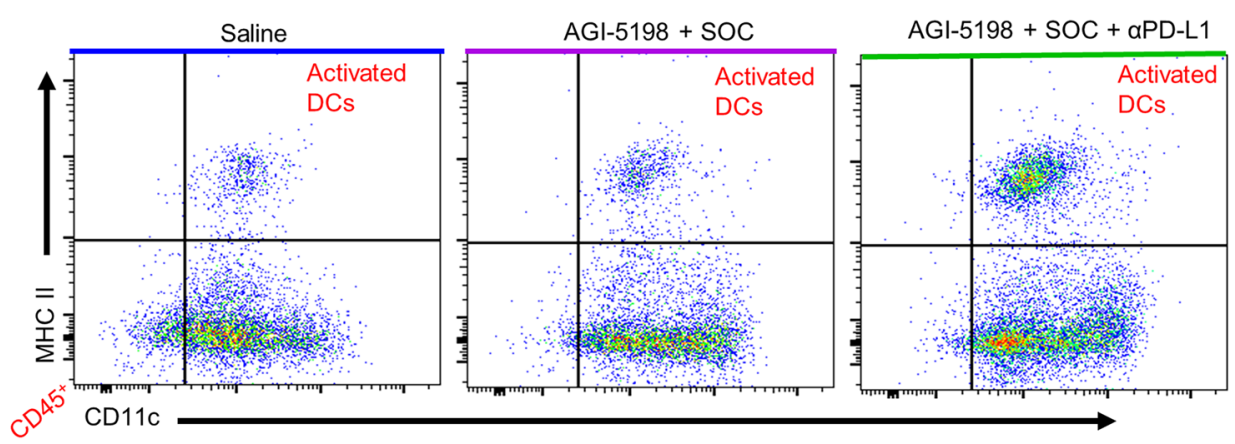

MHC II+ DCs

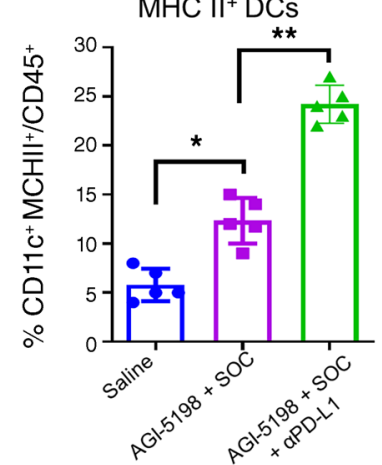

Figure 6. Inhibition of IDH1-R132H in combination with SOC and $\alpha$ PD-L1 leads to higher frequency of dendritic cells (DCs) in the mIDH1 glioma TME. (A) Experimental design to characterize DCs in the TME of mIDH1-OVA glioma-bearing mice after treatment. (B) The percentage of pDCs (CD11 $\left.\mathrm{C}^{+} / \mathrm{B}^{2} 2 \mathrm{O}^{+}\right)$and CDCs (CD11 $\left.\mathrm{C}^{+} / \mathrm{B} 200^{-}\right)$within the CD45+ cell population in the TME of saline-, AGI-5198+IR-, or AGI-5198+SOC+ $\alpha$ PD-L1-treated mIDH1 tumor-bearing mice was assessed at day $27 \mathrm{dpi}$. Representative flow plots for each group are displayed. ${ }^{*} P<0.05$; ${ }^{* *} P<0.01,1$-way ANOVA. Bars represent mean \pm SEM ( $n$ $=5$ biological replicates). (C) Activation status of CD11 ${ }^{+}$DCs in the mIDH1 TME was assessed by expression of MHC II. Representative flow plots for each treatment are displayed. ${ }^{*} P<0.05 ;{ }^{* *} P<0.001$, 1-way ANOVA. Bars represent mean \pm SEM ( $n=5$ biological replicates).

To determine the effect of AGI-5198+SOC+ $\alpha$ PD-L1 treatment on DC activation status, we measured expression levels of costimulatory molecules CD80, CD86, and MHC II (26-28) in the DCs of the mIDH1 TME. We observed an increase in MHC II (approx- imately 2.4-fold, $P \leq 0.05)$, CD80 (0.8-fold, $P \geq 0.05$ ), and CD86 (approximately 2.0 -fold, $P \leq 0.05) \mathrm{CD}^{2} 5^{+} / \mathrm{CD} 11 \mathrm{c}^{+}$DCs positive cells in the TME of AGI-5198+SOC-treated mice when compared with saline-treated mice (Figure 6C, and Supplemental Figure 19). 
A
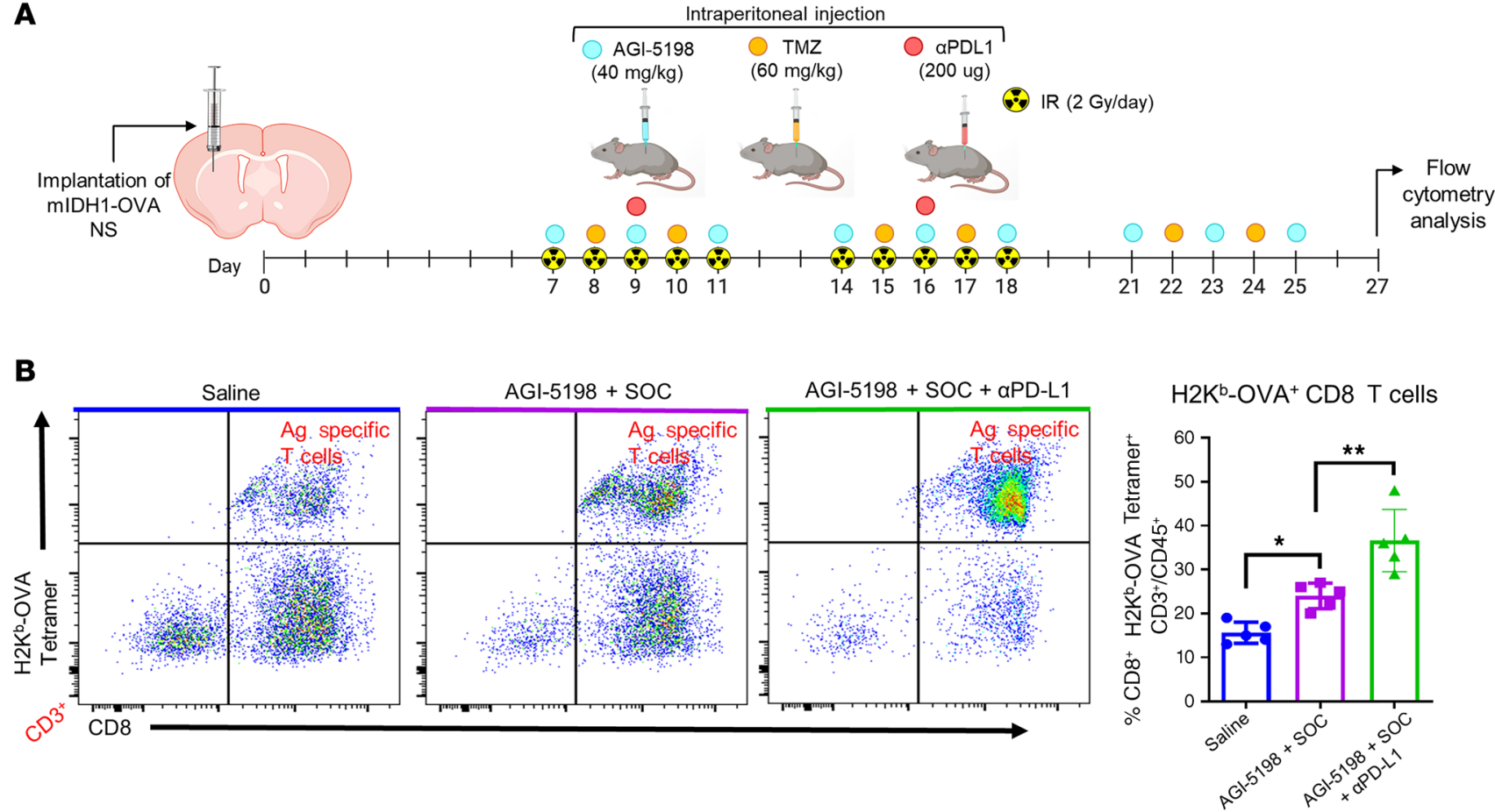

C
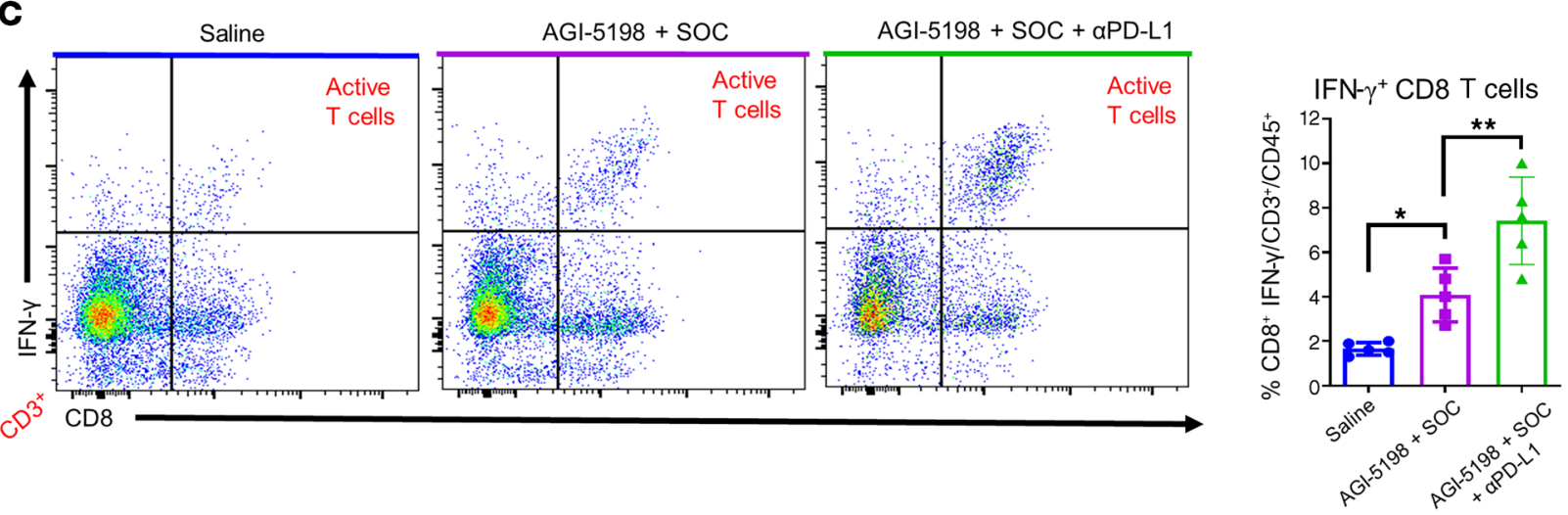

Figure 7. Inhibition of IDH1-R132H in combination with SOC and $\alpha$ PD-L1 induces tumor antigen-specific CD8 T cell responses within mIDH1 glioma TME. (A) Experimental design to characterize T cells in the TME of mIDH1-OVA glioma-bearing mice after treatment. (B) Tumor-specific CD8 ${ }^{+}$T cells within the TME of mIDH1-OVA tumors were analyzed by staining with the SIINFEKL-Kb tetramer. Representative flow plots for each treatment are displayed. ${ }^{* *} P<$ $0.01 ;{ }^{* *} P<0.001$, 1-way ANOVA. (C) Activation status of $C D 8^{+} T$ cells within the TME of mIDH1-OVA tumors was analyzed by staining for IFN- $\gamma$ after TME stimulation with the tumor cell lysate. ${ }^{* *} P<0.01 ;{ }^{* * *} P<0.001$, 1-way ANOVA. Bars represent mean \pm SEM $(n=5$ biological replicates).

The activation status of DCs was further enhanced by the addition of $\alpha$ PD-L1 to AGI-5198+SOC treatment. We observed an increase in the frequency of MHC II (approximately 2.0-fold, $P \leq 0.01$ ), CD80 (approximately 2.1-fold, $P \leq 0.01$ ), and CD86 (approximately 1.8-fold, $P \leq 0.001$ ) DCs in the TME (Figure 6C, and Supplemental Figure 19). These data suggest that AGI-5198+SOC+ $\alpha$ PD-L1 treatment promotes an enhanced adaptive immune response within the mIDH1 glioma TME through the recruitment and activation of antigen-presenting DCs.

To further evaluate the specificity of the anti-mIDH1 glioma immune response elicited by AGI-5198+SOC $+\alpha$ PD-L1 treatment, we assessed antigen-specific $\mathrm{CD}^{+} \mathrm{T}$ cell responses in the TME of mIDH1-OVA glioma-bearing mice in each treatment group (Figure 7A). We observed a 1.6-fold $(P \leq 0.05)$ increase in tumor antigen-specific $\mathrm{CD}^{+} / \mathrm{CD}^{+} /$SIINFEKL-H2K $\mathrm{K}^{\mathrm{b}}$ tetramer ${ }^{+}$
T cells in the TME of mIDH1-OVA tumor-bearing mice treated with AGI-5198+SOC compared with the saline treatment group (Figure 7B). This was also enhanced in mice treated with AGI$5198+$ SOC $+\alpha$ PD-L1 by approximately 1.4 -fold $(P \leq 0.01$; Figure $7 \mathrm{~B})$. We observed a significant increase in total $\mathrm{CD}^{+}$and $\mathrm{CD}^{+} /$ $\mathrm{CD}^{+} \mathrm{T}$ cells in the TME of mIDH1-OVA glioma-bearing mice treated with AGI-5198+SOC+ $\alpha$ PD-L1 compared with the saline and AGI-5198+SOC treatment groups (Supplemental Figure 19). No differences were observed in the frequency of $\mathrm{CD}^{+} / \mathrm{CD}^{+} \mathrm{T}$ cells between the 3 treatment groups (Supplemental Figure 20). Blood serum analysis showed that IL-12, a cytokine critical for $\mathrm{CD}^{+}$cytotoxic cell activity (29), increased approximately 1.6-fold $(P \leq 0.001)$ and approximately 2.7 -fold $(P \leq 0.001)$ in mice treated with AGI-5198+SOC $+\alpha$ PD-L1 compared with those treated with AGI-5198+SOC or saline, respectively (Supplemental Figure 21). 
A

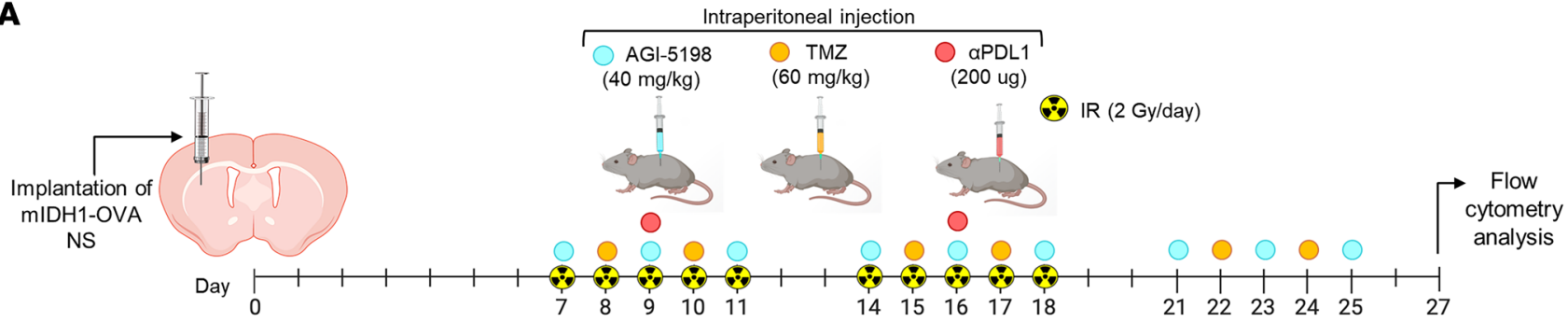

B
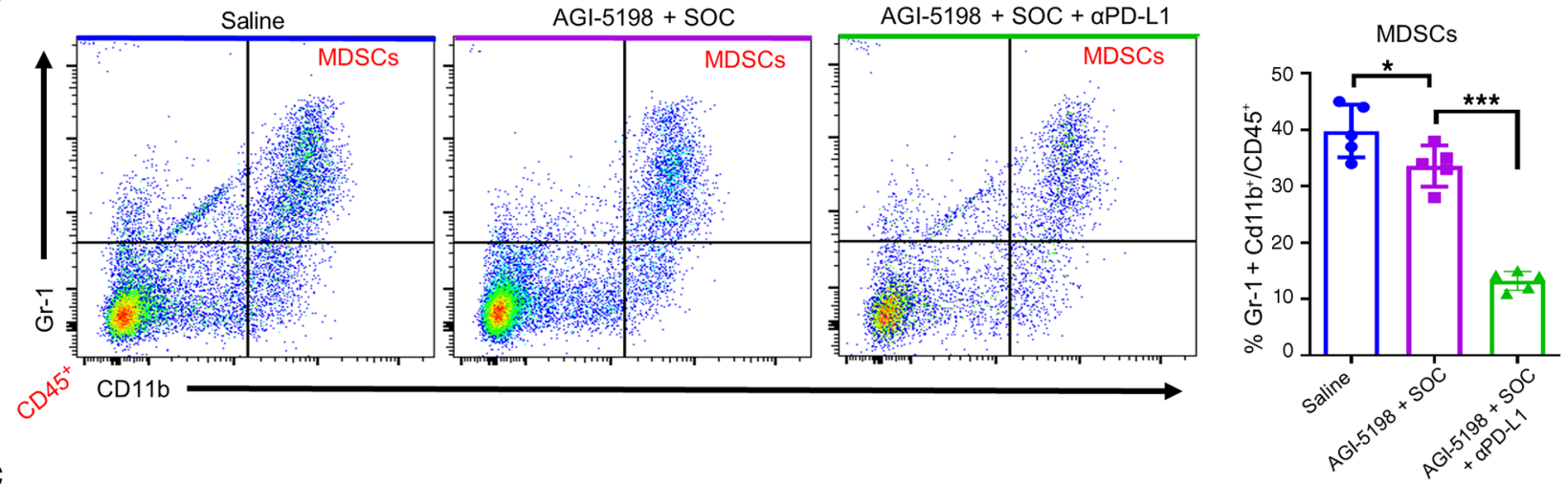

C
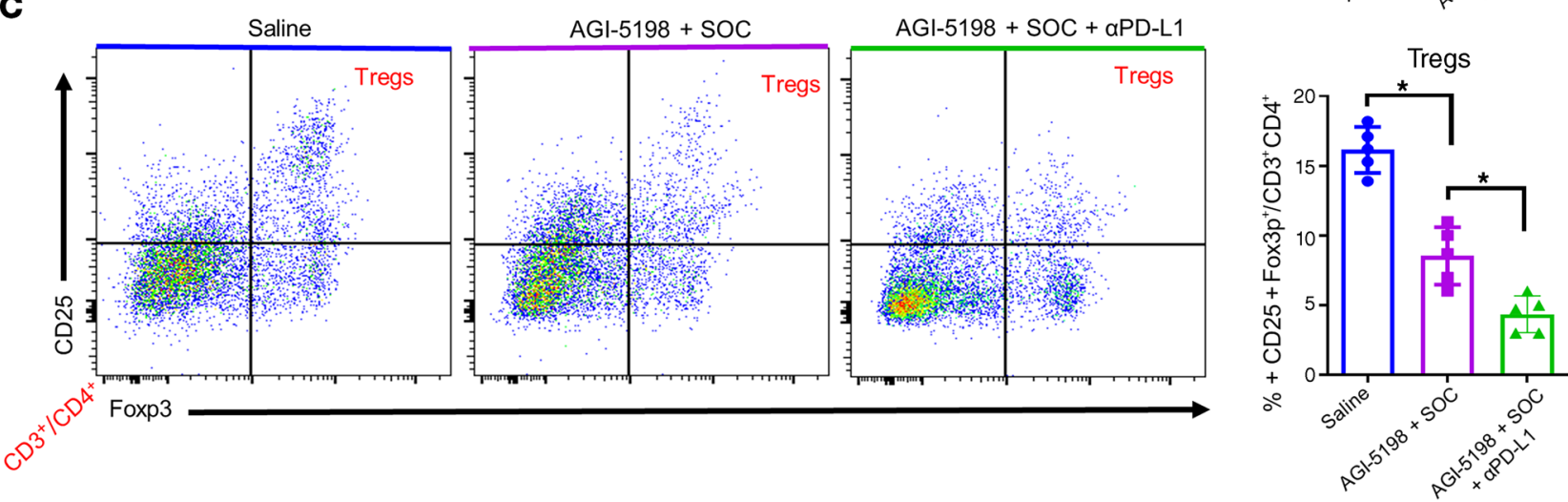

Figure 8. Inhibition of IDH1-R132H in combination with SOC and $\alpha$ PD-L1 decreases the accumulation of MDSCs and Tregs in TME of mIDH1 glioma. (A) Experimental design to characterize MDSCs and Tregs in the TME of mIDH1-OVA glioma-bearing mice after treatment. (B) The percentage of MDSCs $\left(\mathrm{CD} 11 \mathrm{~b}^{+} / \mathrm{Gr} 1^{+}\right)$within the CD45+ cell population in the TME of saline-, AGI-5198+IR-, or AGI-5198+SOC+ $\alpha$ PD-L1-treated mIDH1 glioma-bearing mice was assessed at $27 \mathrm{dpi}$. Representative flow plots for each group are displayed. ${ }^{*} P<0.05 ;{ }^{* *} P<0.001,1$-way ANOVA. Bars represent mean \pm SEM $(n=5$ biological replicates). (C) The percentage of Tregs (CD4+/CD25+/Foxp3 $3^{+}$within the CD45+ cell population in the TME of saline-, AGI-5198+IR-, or AGI-5198+SOC $\alpha \mathrm{PD}-\mathrm{L1}$-treated mIDH1 glioma-bearing mice was assessed at $27 \mathrm{dpi}$. Representative flow plots for each group are displayed. ${ }^{*} P<0.05,1$-way ANOVA. Bars represent mean \pm SEM ( $n=5$ biological replicates)

We also examined the impact of AGI-5198+SOC and AGI$5198+\mathrm{SOC}+\alpha \mathrm{PD}-\mathrm{L} 1$ on the activation status of $\mathrm{CD} 3^{+} / \mathrm{CD} 8^{+} \mathrm{T}$ cells in the TME through quantification of IFN- $\gamma$ expression levels. The IFN- $\gamma$ levels of $\mathrm{CD}^{+} \mathrm{T}$ cells isolated from the TME of mice treated with AGI-5198+SOC were approximately 2 -fold $(P \leq 0.05)$ greater than those of $\mathrm{CD}^{+} \mathrm{T}$ cells isolated from control mice treated with saline (Figure 7C). This response also increased approximately 1.4-fold $(P \leq 0.01)$ in mice treated with AGI-5198+SOC+ $+\alpha$ PD-L1 (Figure 7C). Collectively, these data demonstrate that AGI$5198+$ SOC $+\alpha$ PD-L1 therapy induces a robust antitumor immune response through the expansion and activation of mIDH1 gliomaspecific $\mathrm{CD} 8^{+} \mathrm{T}$ cells.

Treatment of mIDH1 glioma-bearing mice with IDH1-R132H inhibition in combination with SOC and anti-PD-L1 blockade decreases the presence of immunosuppressive cells in the TME. Gliomas have been shown to employ numerous mechanisms to suppress the immune system (30), thus, we evaluated whether AGI5198 , SOC, and $\alpha$ PD-L1 treatment reduces the accumulation of immunosuppressive MDSCs, Tregs, and M2 macrophages (MØ) in the MIDH1 glioma TME. Mice with mIDH1-OVA tumors were treated with saline, AGI-5198+SOC, or AGI-5198+SOC+ $\alpha$ PD-L1. Brains were processed for flow cytometry analysis at $27 \mathrm{dpi}$, as indicated in Figure 8A. We observed a 1.4-fold $(P \leq 0.05)$ decrease in the percentage of tumor-infiltrating MDSCs $\left(\mathrm{CD} 45^{+} / \mathrm{CD} 1 \mathrm{~b}^{+} /\right.$ Gr-1 $1^{+}$) in the AGI-5198+SOC-treated mice compared with the saline-treated mice (Figure 8B). We also observed a 2.3-fold ( $P \leq$ 0.001 ; Figure $8 \mathrm{~B}$ ) decrease in tumor-infiltrating MDSCs in animals treated with AGI-5198+SOC+ $\alpha$ PD-L1 compared with mice 
A

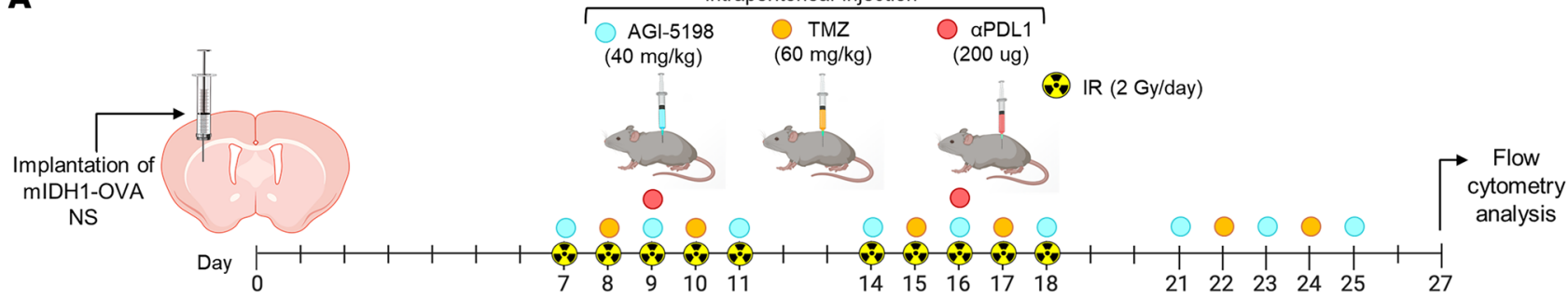

B

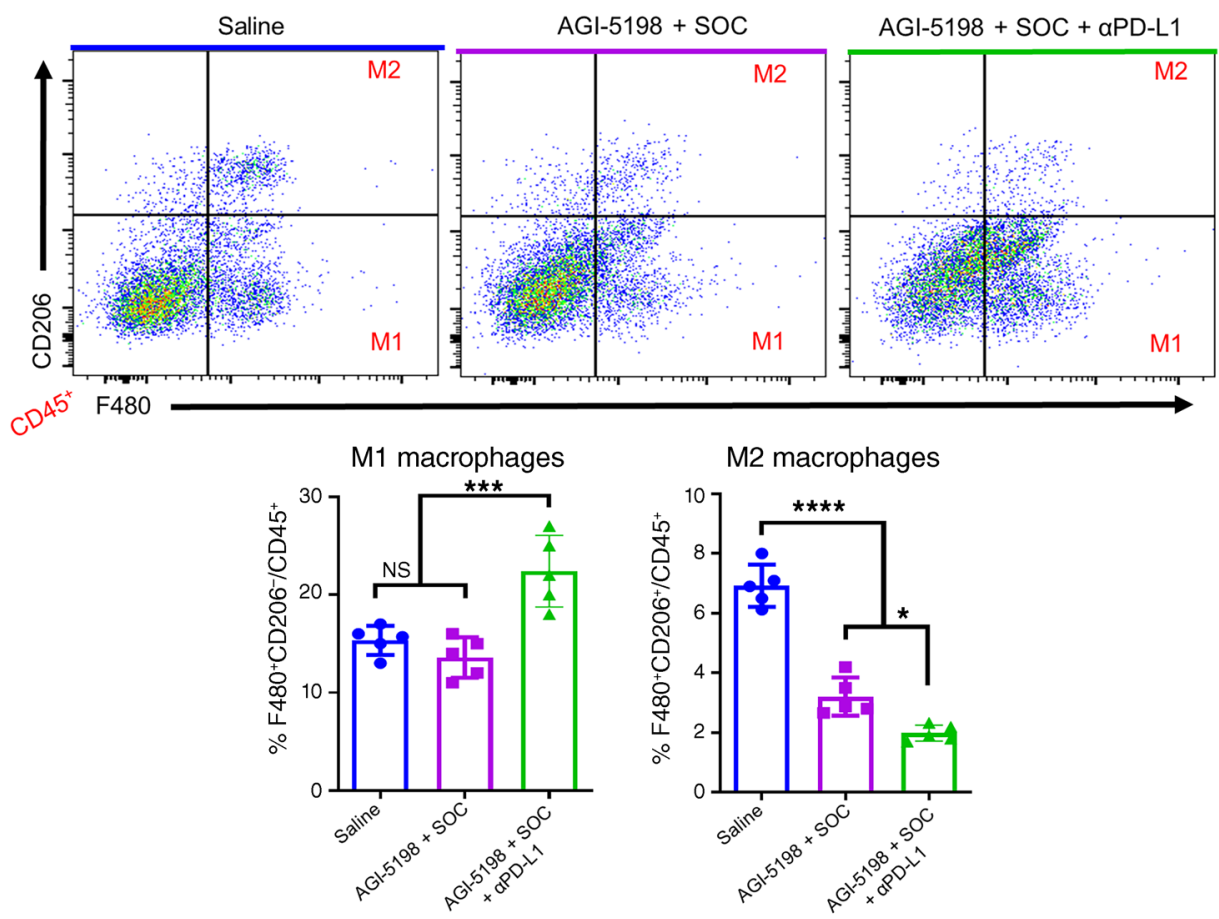

Figure 9. Inhibition of IDH1-R132H in combination with SOC and $\mathrm{PPD}-\mathrm{L1}$ decreases the accumulation of M2 macrophages in TME of mIDH1 glioma. (A) Experimental design to characterize macrophages in the TME of mIDH1-OVA glioma-bearing mice after treatment. (B) The percentage of $\mathrm{M}_{1}$ (F480 ${ }^{+} /$

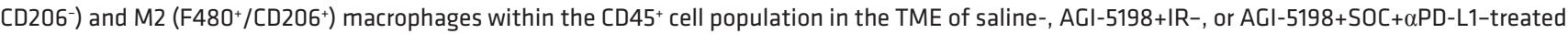
glioma-bearing mice was assessed at $27 \mathrm{dpi}$. Representative flow plots for each group are displayed. ${ }^{*} P<0.05$; ${ }^{* * *} P<0.0001,1$-way ANOVA. Bars represent mean macrophage quantification \pm SEM ( $n=5$ biological replicates).

treated with AGI-5198+SOC. Flow cytometry analysis for Tregs $\left(\mathrm{CD} 5^{+} / \mathrm{CD}^{+} / \mathrm{CD}^{2} 5^{+} / \mathrm{Foxp}^{+}\right)$also showed a decrease in mIDH1 glioma by approximately 2.4 -fold $(P \leq 0.05$; Figure $8 C$ ) in mice treated with AGI-5198+SOC compared with those treated with saline, and an additional decrease of approximately 1.5-fold ( $P \leq$ $0.05)$ in mice treated with AGI-519+SOC $+\alpha \mathrm{PD}-\mathrm{L} 1$ (Figure $8 \mathrm{C}$ ). Moreover, we observed a 1.7-fold $(P \leq 0.0001)$ decrease in the number of tumor-infiltrating M2 MØs $\left(\mathrm{CD}^{2} 5^{+} / \mathrm{F} 480^{+} / \mathrm{CD} 206^{+}\right)$ within the TME of AGI-5198+SOC-treated mice compared with saline-treated mice, and a 1.5 -fold $(P \leq 0.05)$ decrease in mice treated with AGI-519+SOC+ $\alpha$ PD-L1 (Figure 9, A and B). Conversely, we observed a 1.5-fold $(P \leq 0.001)$ increase in the number of M1 MØs within the TME of AGI-5198+SOC+ $\alpha$ PD-L1-treated mice compared with mIDH1 glioma-bearing mice treated with AGI-5198+SOC or saline (Figure 9B). Overall, we observed that AGI-5198+SOC $+\alpha$ PD-L1 treatment significantly decreases the amount of immunosuppressive MDSCs, Tregs, and M2 MØs in the TME of mIDH1 glioma-bearing mice compared with the AGI$5198+$ SOC and saline treatment groups.
Impact of oncometabolite D-2HG on T cell function. D-2HG is produced by mIDH1 glioma cells (9) and released into the MIDH1 glioma TME. Thus, we next aimed to evaluate the impact of $\mathrm{D}-2 \mathrm{HG}$ on $\mathrm{T}$ cell function. Numerous studies have shown D-2HG to be poorly cell permeable (24). We examined the effect of D-2HG on the activation and proliferation status of antigen-specific $\mathrm{CD} 8^{+}$ $\mathrm{T}$ cells from OT- 1 transgenic mice, which have $\mathrm{T}$ cell receptors engineered to recognize the SIINFEKL peptide (31). OT-1 splenocytes were fluorescently labeled with 5- and 6-carboxyfluorescein diacetate succinimidyl ester (CFSE) and stimulated for 4 days with $100 \mathrm{nM}$ SIINFEKL peptide in the presence or absence of $0.25 \mathrm{mM}$ D-2HG (Figure 10A). We used this concentration as we observed that the number of $\mathrm{T}$ cells undergoing apoptosis (approximately $22 \%$ ) were similar to the unstimulated control group (Supplemental Figure 22). Proliferation and activation of T cells was assessed by CFSE dilution analysis and quantification of IFN- $\gamma$ expression levels in the supernatants, respectively. Unstimulated OT-1 T cells did not proliferate and showed low IFN- $\gamma$ levels (Figure 10, B-D). However, almost 100\% of T cells from OT-1 mice under- 
A
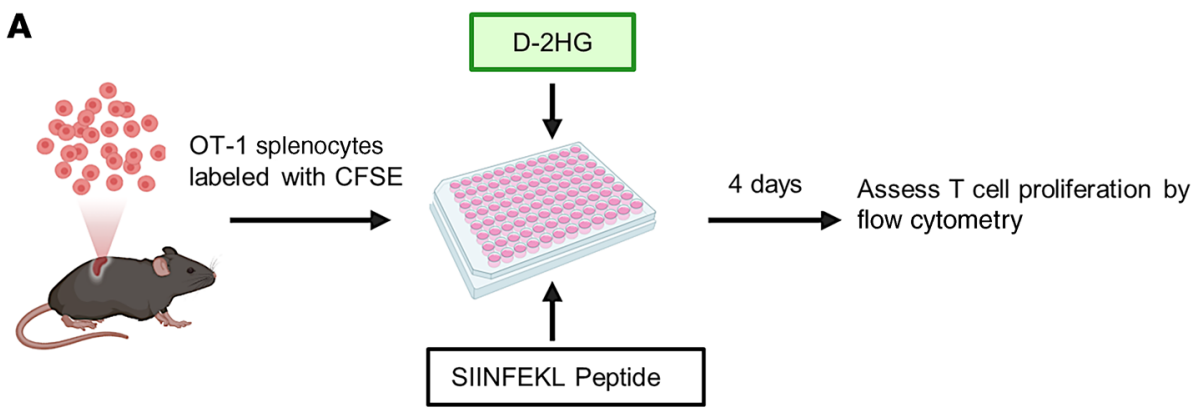

B
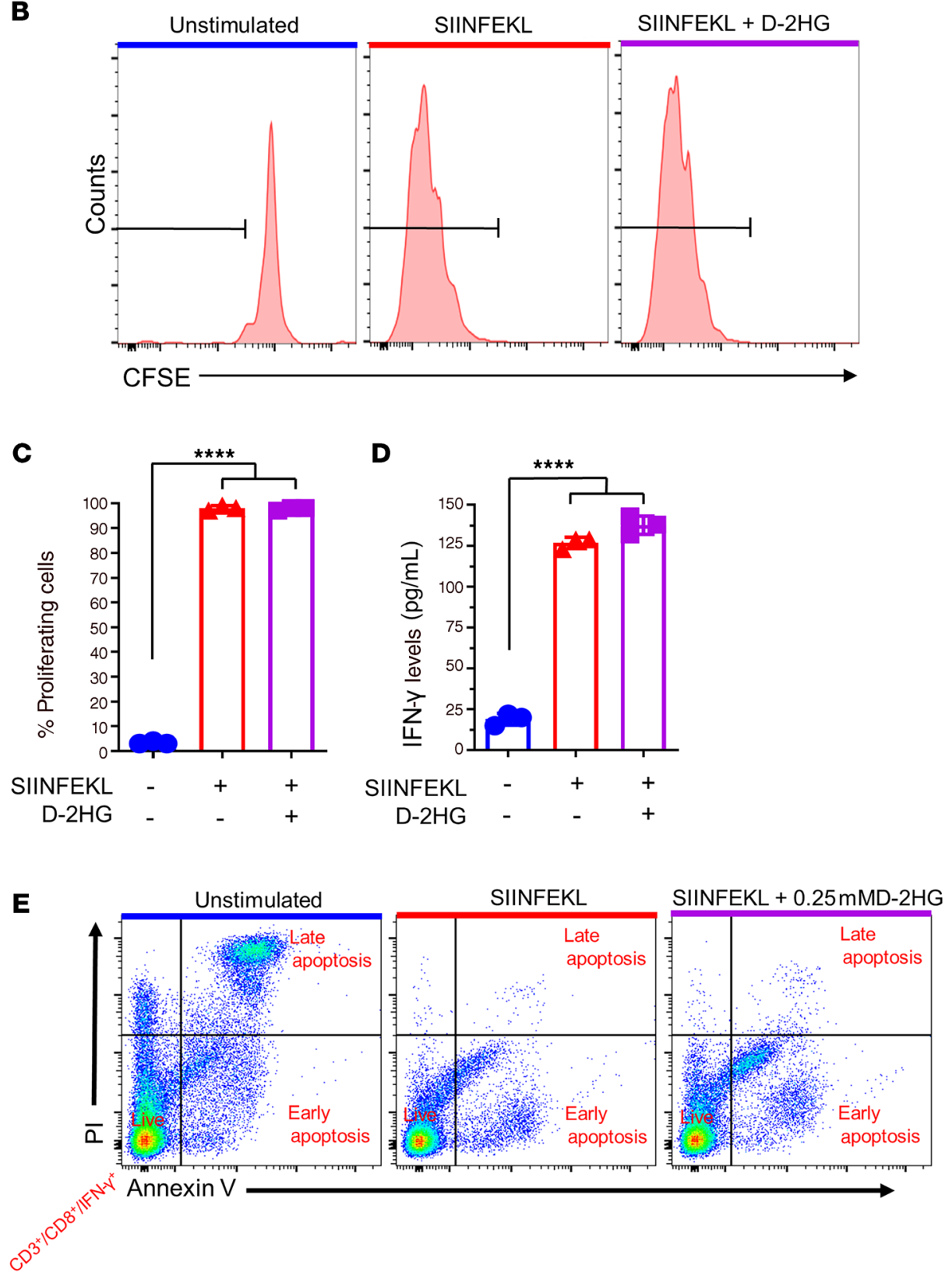

Figure 10. D-2HG does not inhibit T cell proliferation and activation. (A) Experimental design to analyze the impact of D-2HG on $\mathrm{T}$ cell proliferation. (B) Flow plots show representative CFSE stains of unstimulated splenocytes (inactivated T cells), splenocytes undergoing proliferation in response to 100 nM SIINFEKL (activated T cells), and the effect of $0.25 \mathrm{mM} \mathrm{D-2HG}$ on SIINFEKL-induced T cell proliferation. (C) Quantification of OT-1 splenocytes undergoing $T$ cell proliferation. ${ }^{* *} P<0.001,1$-way ANOVA. Bars represent mean \pm SEM ( $n=3$ technical replicates). (D) Quantification of IFN- $\gamma$ levels in the supernatants of OT-1 splenocytes stimulated with SIINFEKL in the presence of D-2HG. IFN- $\gamma$ levels were assessed by ELISA. (E) Representative flow plots of OT-1 splenocytes incubated with $0.25 \mathrm{mM} \mathrm{D-2HG}$ in the presence of $100 \mathrm{nM}$ SIINFEKL peptide for 4 days and stained with Annexin V-FITC and propidium iodide (PI). Live activated $\mathrm{T}$ cells $\left(\mathrm{CD3}^{+} / \mathrm{CDB}^{+} / \mathrm{IFN}-\gamma^{+}\right)$were identified as Annexin $\mathrm{V}$ negative and PI negative. Dead cells undergoing early apoptosis were identified as Annexin $\mathrm{V}$ positive and PI negative. Dead T cells undergoing late apoptosis were identified as Annexin $\mathrm{V}$ positive and PI positive. Bars represent quantitative analysis of the distribution of live and dead cells during 4-day incubation period with D-2HG ( $n=3$ technical replicates).
Live vs. dead T cells

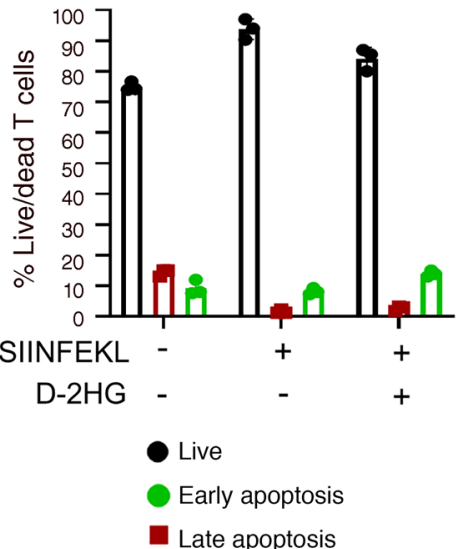


A

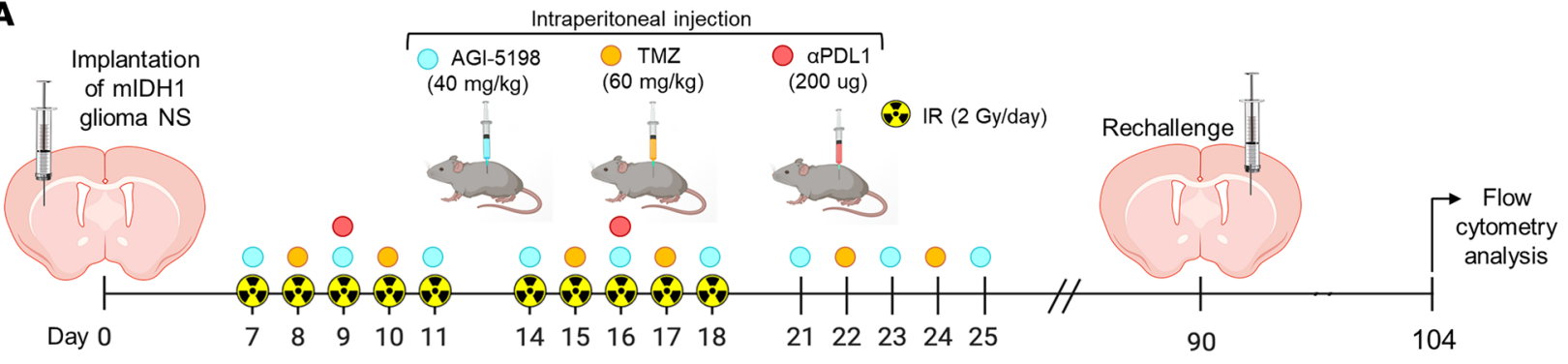

B
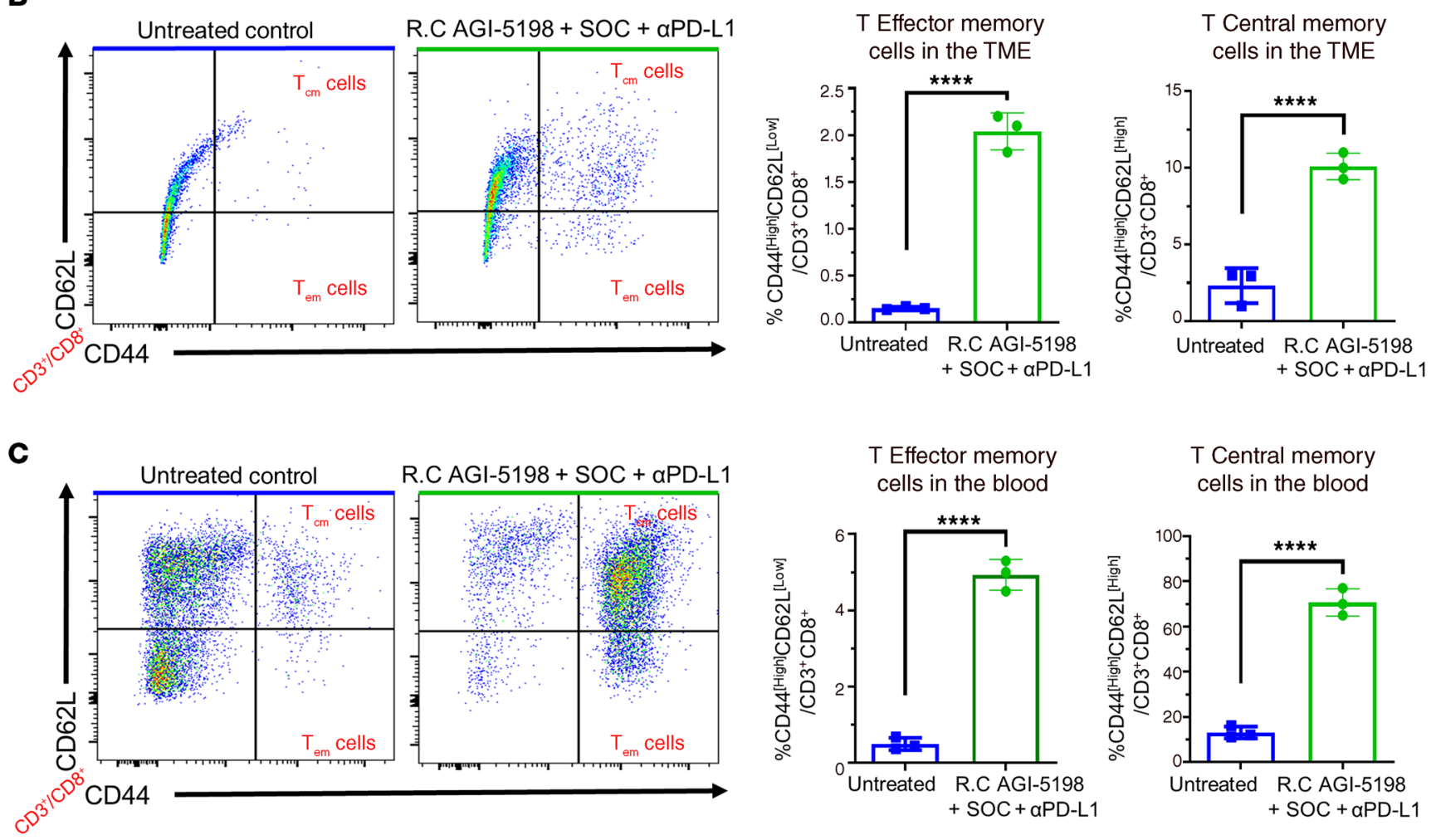

T Central memory cells in the blood
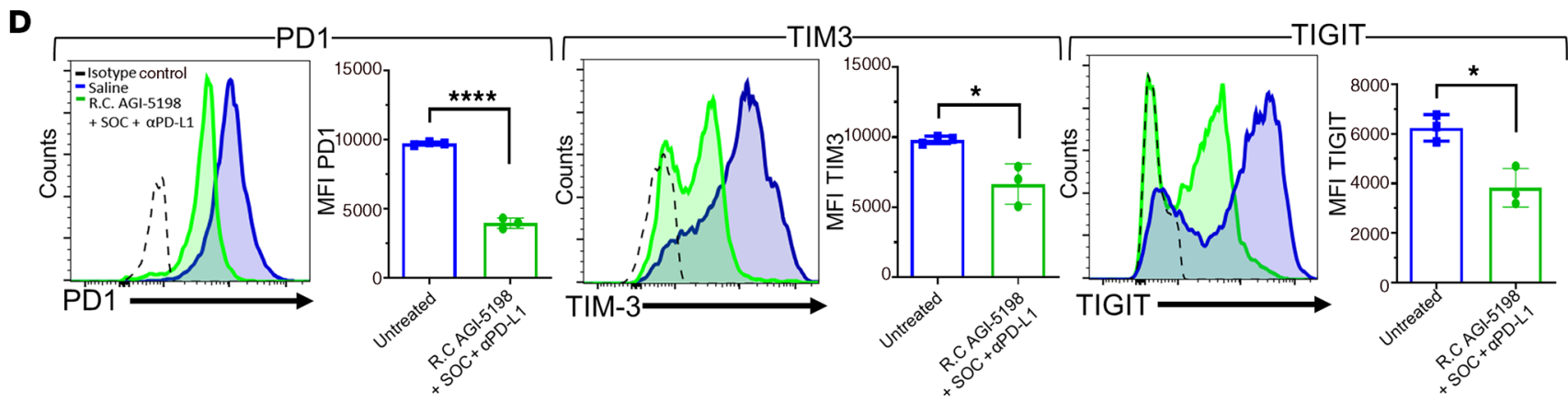

Figure 11. Inhibition of IDH1-R132H in combination with SOC and $\alpha \mathrm{PD}-\mathrm{L} 1$ induces CD8 ${ }^{+} \mathrm{T}$ cell memory responses and lowers $\mathrm{T}$ cell exhaustion within the TME of mIDH1 glioma-bearing mice. (A) Experimental design to assess memory T cell response in mIDH1 glioma-bearing mice treated with

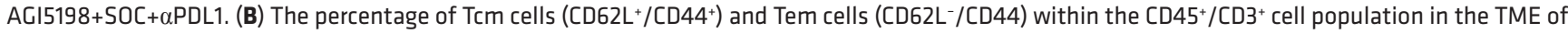
untreated control and AGI-5198+SOC+ $\mathrm{PPD}-\mathrm{L} 1$-treated mice was assessed at $14 \mathrm{dpi}$ after mIDH1 glioma rechallenge (R.C). Representative flow plots for each group are displayed. ${ }^{* * *} P<0.0001$, unpaired, 2-tailed $t$ test. Bars represent mean \pm SEM ( $n=3$ biological replicates). (C) The percentage of Tcm cells $\left(\mathrm{CD}_{2} \mathrm{~L}^{+} / \mathrm{CD} 44^{+}\right)$and Tem cells (CD62L-/CD44) within the $\mathrm{CD} 45^{+} / \mathrm{CD3}^{+}$cell population in the circulation of untreated control and AGI-5198+SOC+ $\alpha \mathrm{PD}-\mathrm{L} 1-$ treated mice was assessed at $14 \mathrm{dpi}$ after mIDH1 glioma rechallenge. Representative flow plots for each group are displayed. ${ }^{* * *} P<0.0001$, unpaired, 2-tailed $t$ test. Bars represent mean \pm SEM ( $n=3$ biological replicates). (D) CD8 $8^{+}$T cell exhaustion in the TME of mIDH1 glioma-bearing mice of untreated control and AGI-5198+SOC+ $\alpha$ PD-L1-treated mice was assessed at 14 days after tumor rechallenge, by assessing the expression levels of PD-1, TIM-3, and TIGIT markers. Representative histograms display each marker's expression levels (black, isotype control; blue, saline; green, AGI-5198+SOC+ $\alpha \mathrm{PD}-\mathrm{L} 1$ ). ${ }^{*} P<$ $0.05 ;{ }^{* * *} P<0.0001$, unpaired, 2-tailed $t$ test. Bars represent mean \pm SEM ( $n=3$ biological replicates). 
went antigen-specific proliferation in response to SIINFEKL stimulation, and IFN- $\gamma$ levels in the supernatant were approximately 5-fold higher than unstimulated splenocytes (Figure 10, B-D). The addition of $\mathrm{D}-2 \mathrm{HG}$ to stimulated $\mathrm{T}$ cells did not significantly alter proliferation of IFN- $\gamma$ levels compared with cells treated with SIINFEKL alone (Figure 10, B-D). These data indicate that D-2HG does not directly impact $\mathrm{T}$ cell activation or proliferation.

Since $\mathrm{T}$ cell functions require viable $\mathrm{T}$ cells, we next evaluated whether D-2HG would induce apoptosis of activated T cells. OT-1 splenocytes were stimulated with $100 \mathrm{nM}$ SIINFEKL peptide in the presence or absence of $0.25 \mathrm{mM} \mathrm{D}-2 \mathrm{HG}$ for 4 days (Figure 10A). Activated $\mathrm{T}$ cells $\left(\mathrm{CD}^{+} / \mathrm{CD}^{+} / \mathrm{IFN}-\gamma^{+}\right)$undergoing early or late apoptosis were identified by flow cytometry with Annexin V/PI staining. In accordance with our data on $\mathrm{T}$ cell activation and proliferation, we observed approximately 90\% live (Annexin $\left.\mathrm{V}^{-} / \mathrm{PI}^{-}\right) \mathrm{T}$ cells in culture with SIINFEKL alone compared with approximately $76 \%$ live $\mathrm{T}$ cells in cultures with unstimulated OT- 1 cells (Figure 10E). The addition of D-2HG to the T cell culture with SIINFEKL did not induce apoptosis (Figure 10E). Notably, when nonactivated $\mathrm{T}$ cells were cultured using a range of 5-30 mM D-2HG, we observed that T cell apoptosis increased in a dose-dependent manner (Supplemental Figure 22). These data indicate that at higher doses $\mathrm{D}-2 \mathrm{HG}$ is toxic for $\mathrm{CD}^{+} \mathrm{T}$ cells, rather than inhibiting their proliferating capacity. Overall, our data indicate that $\mathrm{D}-2 \mathrm{HG}$, which is present in the mIDH1 glioma TME, does not alter $\mathrm{T}$ cell function.

IDH1-R132H inhibition in combination with SOC and antiPD-L1 immune checkpoint blockade confers memory T cells' response following glioma rechallenge. We demonstrated that $\mathrm{mIDH} 1$ glioma-bearing mice survive long term when treated with AGI$5198+$ SOC $+\alpha$ PD-L1 and that long-term survivors exhibit immunological resistance to mIDH1 glioma rechallenge in the contralateral hemisphere (Figure 5, C and D). Therefore, we assessed the $\mathrm{T}$ cell memory response in the rechallenged long-term survivors previously treated with AGI-5198+SOC $+\alpha$ PD-L1 (Figure 11A). Normal untreated mice implanted with $\mathrm{mIDH} 1$ glioma were utilized as controls. Mice were euthanized 14 days after rechallenge, brains and blood were processed for flow cytometry analysis of $\mathrm{CD} 5^{+} / \mathrm{CD}^{+} / \mathrm{CD}^{+} / \mathrm{CD} 44^{\text {hi }} / \mathrm{CD} 2 \mathrm{~L}^{\text {lo }}$ effector (Tem) and $\mathrm{CD} 5^{+} /$ $\mathrm{CD}^{+} / \mathrm{CD}^{+} / \mathrm{CD} 44^{\text {hi }} / \mathrm{CD} 6 \mathrm{~L}^{\text {hi }}$ central $(\mathrm{Tcm})$ memory $\mathrm{T}$ cells. Mice treated with AGI-5198+SOC+ $\alpha$ PD-L1 showed an increase in the percentage of TME infiltrating Tem (approximately 8-fold; $P \leq 0.0001$ ) and Tcm (approximately 3.3-fold; $P \leq 0.0001$ ) cells compared with the untreated control mice (Figure 11B). We also observed an increase in the percentage of Tem (approximately 1.6-fold; $P \leq 0.0001$ ) and Tcm (approximately 7-fold; $P \leq 0.0001$ ) cells in the blood of mice treated with AGI-5198+SOC $+\alpha$ PD-L1 compared with the control mice (Figure 11C). Blood serum analysis showed that IL-15, a cytokine critical for $\mathrm{CD} 8^{+}$memory $\mathrm{T}$ cell activity $(32)$, increased approximately 4 -fold $(P \leq 0.001)$ in mice treated with AGI-5198+SOC $+\alpha$ PD-L1 compared with untreated mice (Supplemental Figure 23).

Since $\alpha$ PD-L1 may augment the effector functions of gliomainfiltrating cytotoxic $\mathrm{T}$ cells, we tested the effect of AGI$5198+$ SOC $+\alpha$ PD-L1 treatment on T cell exhaustion by measuring the expression levels of PD-1, TIM-3, and TIGIT markers (33) on the $\mathrm{CD}^{+} \mathrm{T}$ cells in the TME of rechallenged mIDH1 glioma-bear- ing mice. We observed a decrease in PD-1 (approximately 3.3-fold, $P \leq 0.001$ ), TIM-3 (approximately 1.3-fold, $P \leq 0.05$ ), and TIGIT (approximately 0.6-fold, $P \leq 0.05$ ) expression on $\mathrm{CD}^{4} 5^{+} / \mathrm{CD}^{+} /$ $\mathrm{CD}^{+} \mathrm{T}$ cells in the TME of rechallenged AGI-5198+SOC+ $\alpha \mathrm{PD}-\mathrm{L} 1-$ treated mice compared with untreated control mice (Figure 11D). These data indicate that the combination of AGI-5198, SOC, and $\alpha \mathrm{PD}-\mathrm{L} 1$ treatment reduces T cell exhaustion and strongly favors the generation of a memory $\mathrm{CD}^{+} \mathrm{T}$ cell response against $\mathrm{mIDH} 1$ glioma. This treatment strategy promotes the generation of adaptive immune response against tumor recurrence.

\section{Discussion}

A critical factor influencing the success of therapeutic approaches for gliomas expressing IDH1-R132H (mIDH1) in the context of TP53 and ATRX inactivation is the ability to harness the mediated responses of memory $\mathrm{T}$ cells in order to prevent recurrence. Several preclinical studies have shown that small molecule inhibitors targeting IDH1-R132H have been effective in hampering tumor progression when used as monotherapy in preclinical models of AML and glioma (17, 19, 24, 34, 35).

We showed that the genetic context in which IDH-R132H mutation occurs impacts tumor biology and treatment outcomes (9). For instance, we demonstrated that D-2HG epigenetically reprograms the expression of genes involved in DNA-damage response in gliomas harboring IDH1-R132H in the context of ATRX and TP53 inactivation (mIDH1), eliciting glioma radioresistance (9). Treatment of mouse and human mIDH1 glioma cells with IDH1-R132H inhibitor in vitro radiosensitized mIDH1 glioma cells. Another study demonstrated that IDH1 glioma radioresistance was determined by the genetic context in which IDH-R132H was expressed (36). These studies suggest that the response to therapies for mutant IDH1 gliomas is dependent on the genetic context in which this mutation is found $(9,36,37)$. Also, ATRX inactivation can have profound effects on chromatin structure, which in turn will affect the transcriptional profile of the cells in which this genetic lesion is encountered (38-40), thus it could impact therapeutic efficacy, including the efficacy of $\alpha$ PD-L1 blockade.

In the clinical setting, new therapeutic approaches are required to be tested in combination with SOC, hence we assessed the impact of inhibiting IDH1-R132H enzymatic activity in combination with IR and TMZ in our preclinical mIDH1 model. We found that the MGMT promoter methylation levels in MIDH1 NS are higher compared with WT-IDH1 NS, leading to lower MGMT expression in mIDH1 mouse-NSs compared with WT-IDH mouseNSs (Supplemental Figure 13). Our in vivo data show that administration of TMZ alone to mice bearing MIDH1 tumor confers a modest survival benefit compared with saline control group. TMZ treatment has been associated with hypermutation and malignancy, downregulation of PD-L1, and lymphopenia in patients with glioma (41-43). A reduction in the circulating levels of $\mathrm{CD}^{+} \mathrm{T}$ cells and an increase in circulating Treg levels has also been observed in patients with glioma receiving systemic $\operatorname{TMZ}(41,44,45)$. We and others demonstrated that although TMZ increases the infiltration of Tregs into the TME, it does not adversely affect the therapeutic efficacy of immunotherapy $(42,43,46,47)$. In addition, TMZ has been shown to not adversely impact antitumor immuni- 
ty when combined with immunotherapies in patients with glioma (41). Furthermore, it is also well documented that TMZ induces the expression of proinflammatory molecules such as calreticulin and HMGB1 by glioma cells, enhancing the immunogenicity of tumor cells $(42,48)$. Our data show that concomitant administration of TMZ with IR, AGI-5198, and $\alpha$ PD-L1 elicits a reduction in the accumulation of immunosuppressive MDSCs, Tregs, and M2 MØs within the mIDH1 glioma TME (Figures 8 and 9), indicating an immune-permissive TME.

A critical feature of any proposed therapeutic strategy for mIDH1 gliomas should be the ability to prevent tumor recurrence, which is a salient clinical feature of this disease $(49,50)$. The establishment of immunological memory after immunotherapy is mediated by tumor-specific memory $\mathrm{T}$ cells that can protect against tumor recurrence (51). A previous study demonstrated that in primary IDH1-R132H grade II/III gliomas there is less infiltration of $\mathrm{CD}^{+} \mathrm{T}$ cells in the TME; $\mathrm{CD} 8^{+} \mathrm{T}$ cells were predominantly localized in the perivascular niche (52). This reduction in $\mathrm{T}$ cell numbers was due to decreased expression of cell adhesion molecules (ICAM1) and chemoattractants (CXCL9 and CXCL10), which mediate the recruitment of $\mathrm{T}$ cells from circulation into the TME (52). Similarly, another study demonstrated less intratumoral infiltration of $\mathrm{CD}^{+} \mathrm{T}$ cells in syngeneic GL261 and SB-derived SB28 (driven by NRAS, PDGF- $\beta$, and short hairpin-targeting TP53) models expressing IDH1-R132H compared with WT-IDH1 tumors. The authors demonstrated reduced IFN- $\gamma$ expression in the T cells present within the IDH1R132H glioma TME (24). Further, reduced expression of genes involved in leukocyte migration was observed in IDH1-R132H tumor-bearing Ntva_Ink4a/Arf ${ }^{+-}$mice (7). However, none of these studies addressed IDH1-R132H in the genetic context of ATRX and TP53 inactivation.

In this study, we aimed to assess the immunological impact of blocking D-2HG production on anti-mIDH1 glioma immunity in our genetically engineered mutant IDH1 mouse glioma model, which also harbors ATRX and TP53 loss, recapitulating the salient genetic lesions encountered in mutant IDH1 astrocytomas $(2,9)$. We demonstrate that systemic administration of an IDH1-R132H inhibitor prolonged the MS of MIDH1 glioma-bearing mice, with $40 \%$ being long-term survivors (Figure 2). Furthermore, when the long-term survivors from the IDH1-R132H inhibition treatment group were rechallenged with $\mathrm{mIDH} 1$ tumor cells in the contralateral hemisphere, all the mice remained tumor free without further treatment, indicating the development of anti-glioma immunological memory (Figure 2). The reason that $40 \%$ of the mice treated with AGI-5198 were tumor free could be due to the host's ability to mount a robust effector immune response, unlike the $60 \%$ of the mice which developed therapeutic resistance and succumbed due to tumor burden. The variability in the response to combination of radiotherapy, chemotherapy, and immunotherapy in brain tumor preclinical models has previously been demonstrated by several research groups (53-57). Mechanisms that contribute to treatment resistance are at present unknown and require further investigation (53-57).

Mutant IDH1 gliomas have been reported to accumulate D-2HG to levels as high as $30 \mathrm{mM}$ (58). A recent study suggests that the $\mathrm{D}-2 \mathrm{HG}$ directly inhibits anti-glioma $\mathrm{CD}^{+} \mathrm{T}$ cell func- tions in mIDH1 glioma TME (59). However, previous reports have shown that D-2HG is poorly cell permeable $(11,60)$. Herein, we demonstrate that D-2HG does not suppress the functions of mouse antigen-specific $\mathrm{CD}^{+} \mathrm{T}$ cell proliferation (Figure 10). When we cultured $\mathrm{CD} 8^{+} \mathrm{T}$ cells with $\mathrm{D}-2 \mathrm{HG}$ at concentrations ranging from 5-30 $\mathrm{mM}$, we found that $\mathrm{D}-2 \mathrm{HG}$ induced apoptosis in a dose-dependent manner. Around $40 \%$ of $\mathrm{CD}^{+} \mathrm{T}$ cells exhibited apoptosis when treated with $5 \mathrm{mM} \mathrm{D}-2 \mathrm{HG}$, increasing to more than $70 \%$ apoptosis when treated with $30 \mathrm{mM} \mathrm{D}-2 \mathrm{HG}$ (Supplemental Figure 22). These results indicate that the doses previously reported might have been toxic for $\mathrm{CD} 8^{+} \mathrm{T}$ cells, rather than inhibiting their proliferation.

Immunogenic cell death (ICD) elicited by dying tumor cells contributes to the development of adaptive immunity (61). Our results show that blocking D-2HG production in mouse and human mIDH1 glioma increases the expression of CRT, the release of ATP, and the level of HMGB1 DAMPs molecules (Figure 1). Although we have shown that mIDH1 mouse and human glioma cells are radioresistant (9), we observed that mIDH1 cells treated with IR released ATP at levels similar to those in the IDH1R32H inhibition treatment group (Figure 1), causing activation of the autophagy pathway for survival (21-23) (Supplemental Figure 1). In this respect, it has been shown that tumor cells treated with radiation release ATP, which triggers autophagy activation (21-23).

ICD elicits the activation of antigen-presenting DCs to prime cytotoxic $\mathrm{CD}^{+} \mathrm{T}$ cells and leads to a robust anti-mIDH1 glioma response in our model. Mice bearing $\mathrm{MIDH} 1$ glioma treated with IDH1-R132H inhibitor in combination with SOC demonstrated an increase in pDCs and cDCs in the TME compared with mice in the saline-treated group. This treatment strategy also upregulated the expression of costimulatory ligands CD80, CD86, and MHC II on DCs (26-28), polarizing them toward an activated phenotype (Figure 6, and Supplemental Figure 19). In addition, using a mIDH1 mouse glioma model harboring surrogate tumor antigen ovalbumin (OVA), we monitored the generation of mIDH1 gliomaspecific $\mathrm{T}$ cells in the TME using the $\mathrm{H} 2 \mathrm{~K}^{\mathrm{b}}$ tetramer. Our data show an increase in mIDH1 glioma-specific T cells in the TME of MIDH1 glioma-bearing mice treated with IDH1-R132H inhibitor and SOC compared with mice treated with saline (Figure 7). We observed that the percentage of tumor-specific $\mathrm{CD}^{+} \mathrm{T}$ cells is greater than IFN- $\gamma^{+} \mathrm{T}$ cells in both AGI-5198+SOC or AGI-5198+SOC+ $\alpha$ PD-L1 treatment groups (Figure 7). This could be due to increased proliferation or decreased apoptosis of the tumor antigen-specific $\mathrm{CD}^{+} \mathrm{T}$ cells, which does not indicate that they are exhausted or dysfunctional. The lower percentage of IFN $-\gamma^{+} \mathrm{T}$ cells with respect to the total number of antigen-specific $\mathrm{CD}^{+} \mathrm{T}$ cells in the $\mathrm{mIDH} 1$ glioma microenvironment indicates that not all of the $\mathrm{CD}^{+} \mathrm{T}$ cells are active at a given time point. These data strongly suggest that inhibiting IDH-R132H in combination with SOC induces a mIDH1 glioma-specific immune response.

Immunotherapy has emerged as a powerful therapeutic tool for cancer; however, in glioma it has failed partly due to the presence of inhibitory receptor molecules on T cells, such as programmed cell death protein 1 (PD-1) (30). A study evaluating the immunological gene profile of 282 primary IDH1-R132H gliomas compared with 151 WT-IDH gliomas from TCGA determined that the gene and protein expression of PD-L1 was substantially 
lower in mutant IDH1 gliomas compared with WT-IDH1 glioma (25). We analyzed the expression of PD-L1 in the TCGA data set of WT-IDH, IDH1-R132H with 1p/19q codeletion, and IDH1$\mathrm{R} 132 \mathrm{H}$ with ATRX/TP53 mutation in grade II and III gliomas. We observed that IDH-R132H is associated with lower levels of PD-L1 expression in glioma samples with $1 \mathrm{p} / 19 \mathrm{q}$ codeletion and ATRX/ TP53 mutations (Figure 4A). TCGA analysis also revealed that IDH1-R132H increased DNA methylation at $3 \mathrm{CpG}$ sites within the PD-L1 promoter in $1 \mathrm{p} / 19 \mathrm{q}$ codeleted and $1 \mathrm{p} / 19 \mathrm{q}$ non-codeleted grade II and III gliomas (Figure 4B). This observation is also supported by Mu et al. (25). In this study, we demonstrate that mIDH1 mouse glioma cells in situ exhibit lower levels of PD-L1 compared with WT-IDH mouse glioma cells (Figure 3B). We observed that in vivo inhibition of IDH-R132H leads to increased PD-L1 expression on mIDH1 glioma cells in the TME by approximately 2-fold compared with untreated control mice (Figure 3B). Also, when mIDH1 mouse-NSs and human-GCs were treated with AGI-5198 in vitro, they displayed approximately 9 -fold $(P \leq 0.01)$ lower PD-L1 promoter methylation levels relative to vehicle control (Figure 3E, and Figure 4C). Our results show that inhibition of IDH1-R132H reverses PD-L1 promoter methylation, leading to enhanced PD-L1 expression on mIDH1 glioma cells.

Since higher expression of PD-L1 on tumor cells leads to T cell exhaustion (33) and negatively regulates $\mathrm{T}$ cell activation, priming, and expansion (62), we decided to combine inhibition of D-2HG production with immune checkpoint blockade using $\alpha$ PD-L1. We observed the highest percentage of long-term survivors $(60 \%)$ in the AGI-5198+SOC+ $\alpha$ PD-L1 group compared with all other treatment groups. Our data suggest that adding $\alpha \mathrm{PD}-\mathrm{L} 1$ could enhance the efficacy of AGI-5198+SOC, although the effect estimates did not reach statistical significance due to small sample size. The efficacy of PD-1/PD-L1 immune checkpoint blockade has not been investigated in glioma models harboring IDH1-R132H in the context of ATRX and TP53 loss. We observed that $\alpha \mathrm{PD}-\mathrm{L} 1$, when used as monotherapy, elicited a modest increase in MS of mice bearing $\mathrm{mIDH} 1$ glioma, with no longterm survivors (Figure 4B). There is evidence that shows that immune-checkpoint blockade used as monotherapy has failed in phase III clinical trials to improve overall survival of patients with glioma (20). Our data demonstrate that coadministering $\alpha$ PD-L1 with IDH1-R132H inhibitor and SOC improved the MS of mIDH1 glioma-bearing mice (Figure 5C). Additionally, this strategy reduced $\mathrm{T}$ cell exhaustion and favored the generation of memory $\mathrm{CD}^{+} \mathrm{T}$ cells, which elicited a strong immunological memory response against mIDH1 glioma rechallenge (Figure 11, $\mathrm{B}$ and $\mathrm{C}$ ). Effective antitumor immunity in glioma-bearing mice can be blunted with increased age $(53,54)$. Although we did not age match the untreated control mice (approximately 8 weeks old) with the long-term survivors (17-19 weeks old) in the rechal- lenge study, we observed that an effective memory response was mounted in rechallenged long-term survivors from the AGI $5198+$ SOC $+\alpha$ PDL1 treatment group.

The role of memory $\mathrm{T}$ cells in promoting immunological memory after immunotherapy is critical for preventing mIDH1 glioma recurrence. Our proposed strategy consisting of combining D-2HG inhibition, SOC, and $\alpha \mathrm{PD}-\mathrm{L} 1$ immune checkpoint blockade prolongs MS, yields long-term survivors, and elicits anti-mIDH1 glioma immunological memory (Figure 5, Figure 7, and Figure 11). Therefore, our preclinical data provide evidence that supports the development of combining IDH1-R132H inhibition with SOC and $\alpha$ PD-L1 in a phase I clinical trial for glioma patients expressing IDH1-R132H in the context of TP53, ATRX inactivating mutations.

\section{Methods}

Additional details on the methods are provided in the Supplemental Materials.

Study approval. All studies were approved by and in compliance with the IACUC of the University of Michigan.

\section{Author contributions}

PK, SVC, JCG, MBGF, SH, MSA, FJN, YL, MY, AT, FMN, DL, and MBE performed experiments. PK, JCG, SH, MSA, FJN, YL, CGK, HA, YS, LZ, AS, PRL, and MGC analyzed the data. PK, JCG, YL, AS, PRL, and MGC designed the figures. PK, SVC, JCG, MGF, FJN, FMN, YL, JJM, AS, PRL, and MGC designed the research and contributed to writing and editing the manuscript.

\section{Acknowledgments}

This work was supported by the NIH/National Institute of Neurological Disorders \& Stroke grants R21-NS091555, R37-NS094804, and R01-NS074387 (to MGC) and R01-NS076991, R01-NS082311, and R01-NS096756 (to PRL), and the NIH/National Institute of Biomedical Imaging and Bioengineering grant R01-EB022563 (to PRL and MGC). This work was also supported by the Department of Neurosurgery; Rogel Comprehensive Cancer Center; the Pediatric Brain Tumor Foundation; the American Brain Tumor Association (to MBGF); Leah's Happy Hearts; and Chad Tough Foundations. Experimental design schematics were made using www.biorender.com.

Address correspondence to: Maria G. Castro, University of Michigan Medical School, Department of Neurosurgery, 1150 West Medical Center Drive, MSRB II, Room 4570, Ann Arbor, Michigan 48109, USA. Phone: 734.764.0850; Email: mariacas@med. umich.edu.

FJN's present address is: Leloir Institute Foundation, Buenos Aires, Argentina.
1. Hadziahmetovic M, et al. Recent advancements in multimodality treatment of gliomas. Future Oncol. 2011;7(10):1169-1183.

2. Ceccarelli M, et al. Molecular profiling reveals biologically discrete subsets and pathways of progression in diffuse glioma. Cell. 2016;164(3):550-563.
3. Louis DN, et al. The 2016 World Health Organization classification of tumors of the central nervous system: a summary. Acta Neuropathol. 2016;131(6):803-820.

4. Iorgulescu JB, et al. The misclassification of diffuse gliomas: rates and outcomes. Clin Cancer Res. 2019;25(8):2656-2663.
5. Robinson C, Kleinschmidt-DeMasters BK IDH1-mutation in diffuse gliomas in persons age 55 years and over. J Neuropathol Exp Neurol. 2017;76(2):151-154

6. Reiter-Brennan C, et al. The effects of 2hydroxyglutarate on the tumorigenesis of gliomas. Contemp Oncol (Pozn). 2018;22(4):215-222. 
7. Amankulor NM, et al. Mutant IDH1 regulates the tumor-associated immune system in gliomas. Genes Dev. 2017;31(8):774-786.

8. Brat DJ, et al. Comprehensive, integrative genomic analysis of diffuse lower-grade gliomas. $N$ Engl Med. 2015;372(26):2481-2498.

9. Nunez FJ, et al. IDH1-R132H acts as a tumor suppressor in glioma via epigenetic up-regulation of the DNA damage response. Sci Transl Med. 2019;11(479):eaaq1427.

10. Dang L, et al. Cancer-associated IDH1 mutations produce 2-hydroxyglutarate. Nature. 2009;462(7274):739-744.

11. Xu W, et al. Oncometabolite 2-hydroxyglutarate is a competitive inhibitor of $\alpha$-ketoglutarate-dependent dioxygenases. Cancer Cell. 2011;19(1):17-30.

12. Figueroa ME, et al. Leukemic IDH1 and IDH2 mutations result in a hypermethylation phenotype, disrupt TET2 function, and impair hematopoietic differentiation. Cancer Cell. 2010;18(6):553-567.

13. Chowdhury R, et al. The oncometabolite 2-hydroxyglutarate inhibits histone lysine demethylases. EMBO Rep. 2011;12(5):463-469.

14. Kaartinen V, et al. Cardiac outflow tract defects in mice lacking ALK2 in neural crest cells. Development. 2004;131(14):3481-3490.

15. Turcan S, et al. IDH1 mutation is sufficient to establish the glioma hypermethylator phenotype. Nature. 2012;483(7390):479-483.

16. Urban DJ, et al. Assessing inhibitors of mutant isocitrate dehydrogenase using a suite of pre-clinical discovery assays. Sci Rep. 2017;7(1):12758.

17. Rohle D, et al. An inhibitor of mutant IDH1 delays growth and promotes differentiation of glioma cells. Science. 2013;340(6132):626-630.

18. Popovici-Muller J, et al. Discovery of AG-120 (Ivosidenib): a first-in-class mutant IDH1 inhibitor for the treatment of IDH1 mutant cancers. ACS Med Chem Lett. 2018;9(4):300-305.

19. Golub D, et al. Mutant isocitrate dehydrogenase inhibitors as targeted cancer therapeutics. Front Oncol. 2019;9:417.

20. Krysko DV, et al. Immunogenic cell death and DAMPs in cancer therapy. Nat Rev Cancer. 2012;12(12):860-875.

21. Hou W, et al. Strange attractors: DAMPs and autophagy link tumor cell death and immunity. Cell Death Dis. 2013;4:966.

22. Ko A, et al. Autophagy inhibition radiosensitizes in vitro, yet reduces radioresponses in vivo due to deficient immunogenic signalling. Cell Death Differ. 2014;21(1):92-99.

23. Koukourakis MI, et al. Therapeutic interactions of autophagy with radiation and temozolomide in glioblastoma: evidence and issues to resolve. Br J Cancer. 2016;114(5):485-496.

24. Kohanbash $\mathrm{G}$, et al. Isocitrate dehydrogenase mutations suppress STAT1 and CD8+ T cell accumulation in gliomas. J Clin Invest. 2017;127(4):1425-1437.

25. $\mathrm{Mu} \mathrm{L}$, et al. The IDH1 mutation-induced oncometabolite, 2-Hydroxyglutarate, may affect DNA methylation and expression of PD-L1 in gliomas. Front Mol Neurosci. 2018;11:82.

26. Mineharu Y, et al. Blockade of mTOR signaling via rapamycin combined with immunotherapy augments antiglioma cytotoxic and memory T-cell functions. Mol Cancer Ther. 2014;13(12):3024-3036.

27. Vasilevko V, et al. CD80 (B7-1) and CD86 (B7-2) are functionally equivalent in the initiation and maintenance of CD4+ T-cell proliferation after activation with suboptimal doses of PHA. DNA Cell Biol. 2002;21(3):137-149.

28. Kitamura T, et al. Expression, regulation and function of the costimulatory molecules B7-1(CD80) and B7-2 (CD86) and MHC class II on human enterocytes ${ }^{\dagger} 30$ [preprint]. https://www.biorxiv. org/content/10.1101/2020.05.11.086371v1.full. pdf. Posted on bioRxiv May 12, 2020.

29. Tugues S, et al. New insights into IL-12mediated tumor suppression. Cell Death Differ. 2015;22(2):237-246

30. Quail DF, Joyce JA. The microenvironmental landscape of brain tumors. Cancer Cell. 2017;31(3):326-341.

31. Kamran N, et al. Immunosuppressive myeloid cells' blockade in the glioma microenvironment enhances the efficacy of immune-stimulatory gene therapy. Mol Ther. 2017;25(1):232-248.

32. Pilipow K, et al. IL15 and T-cell stemness in T-cell-based cancer immunotherapy. Cancer Res. 2015;75(24):5187-5193.

33. Lu X, et al. Co-inhibition of TIGIT, PD1, and Tim3 reverses dysfunction of Wilms tumor protein-1 (WT1)-specific CD8+ T lymphocytes after dendritic cell vaccination in gastric cancer. Am J Cancer Res. 2018;8(8):1564-1575.

34. Chaturvedi A, et al. Pan-mutant-IDH1 inhibito BAY1436032 is highly effective against human IDH1 mutant acute myeloid leukemia in vivo. Leukemia. 2017;31(10):2020-2028.

35. Tateishi K, et al. Extreme vulnerability of IDH1 mutant cancers to NAD+ depletion. Cancer Cell. 2015;28(6):773-784

36. Garrett M, et al. Metabolic characterization of isocitrate dehydrogenase (IDH) mutant and IDH wildtype gliomaspheres uncovers cell type-specific vulnerabilities. Cancer Metab. 2018;6:4.

37. Subramanian A, et al. Gene set enrichment analysis: a knowledge-based approach for interpreting genome-wide expression profiles. Proc Natl Acad Sci U S A. 2005;102(43):15545-15550.

38. Koschmann C, et al. ATRX loss promotes tumor growth and impairs nonhomologous end joining DNA repair in glioma. Sci Transl Med. 2016;8(328):328ra28.

39. Herceg Z. Epigenetic mechanisms as an interface between the environment and genome. Adv Exp Med Biol. 2016;903:3-15.

40. Koschmann C, et al. Mutated chromatin regulatory factors as tumor drivers in cancer. Cancer Res. 2017;77(2):227-233.

41. Fadul CE, et al. Immune modulation effects of concomitant temozolomide and radiation therapy on peripheral blood mononuclear cells in patients with glioblastoma multiforme. Neuro Oncol. 2011;13(4):393-400.

42. Candolfi M, et al. Temozolomide does not impair gene therapy-mediated antitumor immunity in syngeneic brain tumor models. Clin Cancer Res. 2014;20(6):1555-1565.
43. Mitchell DA, et al. Monoclonal antibody blockade of IL-2 receptor $\alpha$ during lymphopenia selectively depletes regulatory $\mathrm{T}$ cells in mice and humans. Blood. 2011;118(11):3003-3012.

44. Karachi A, et al. Temozolomide for immunomodulation in the treatment of glioblastoma. Neuro Oncol. 2018;20(12):1566-1572.

45. Brown NF, et al. Harnessing the immune system in glioblastoma. Br J Cancer. 2018;119(10):1171-1181.

46. Desai R, et al. Emerging immunotherapies for glioblastoma. Expert Opin Emerg Drugs. 2016;21(2):133-145.

47. Wainwright DA, et al. Durable therapeutic efficacy utilizing combinatorial blockade against IDO, CTLA-4, and PD-L1 in mice with brain tumors. Clin Cancer Res. 2014;20(20):5290-5301.

48. Kim TG, et al. Immunological factors relating to the antitumor effect of temozolomide chemoimmunotherapy in a murine glioma model. Clin Vaccine Immunol. 2010;17(1):143-153.

49. Leu S, et al. IDH mutation is associated with higher risk of malignant transformation in lowgrade glioma. J Neurooncol. 2016;127(2):363-372.

50. Parsons DW, et al. An integrated genomic analysis of human glioblastoma multiforme. Science. 2008;321(5897):1807-1812.

51. Kamran N, et al. Current state and future prospects of immunotherapy for glioma. Immunotherapy. 2018;10(4):317-339.

52. Weenink B, et al. Low-grade glioma harbors few CD8 T cells, which is accompanied by decreased expression of chemo-attractants, not immunogenic antigens. Sci Rep. 2019;9(1):14643.

53. Ladomersky E, et al. The coincidence between increasing age, immunosuppression, and the incidence of patients with glioblastoma. Front Pharmacol. 2019;10:200.

54. Ladomersky E, et al. IDO1 inhibition synergizes with radiation and PD-1 blockade to durably increase survival against advanced glioblastoma. Clin Cancer Res. 2018;24(11):2559-2573.

55. Curtin JF, et al. HMGB1 mediates endogenous TLR2 activation and brain tumor regression. PLoS Med. 2009;6(1):10.

56. Scheetz L, et al. Synthetic high-density lipoprotein nanodiscs for personalized immunotherapy against gliomas. Clin Cancer Res. 2020;26(16):4369-4380.

57. Hoang-Minh LB, et al. Infiltrative and drugresistant slow-cycling cells support metabolic heterogeneity in glioblastoma. EMBO J. 2018;37(23):98772.

58. Losman JA, et al. What a difference a hydroxyl makes: mutant IDH, (R)-2-hydroxyglutarate, and cancer. Genes Dev. 2013;27(8):836-852.

59 . Bunse L, et al. Suppression of antitumor T cell immunity by the oncometabolite (R)-2-hydroxyglutarate. Nat Med. 2018;24(8):1192-1203.

60. Fu X, et al. 2-Hydroxyglutarate inhibits ATP synthase and mTOR signaling. Cell Metab. 2015;22(3):508-515.

61. Kroemer G, et al. Immunogenic cell death in cancer therapy. Annu Rev Immunol. 2013;31:51-72.

62. Mathios D, et al. Anti-PD-1 antitumor immunity is enhanced by local and abrogated by systemic chemotherapy in GBM. Sci Transl Med. 2016;8(370):370ra180. 